\title{
Loss of the $\mathrm{K}_{\mathrm{v}} 1.1$ potassium channel promotes pathologic sharp waves and high frequency oscillations in in vitro hippocampal slices
}

\author{
Timothy A. Simeone, Ph.D ${ }^{a, b}$, Kristina A. Simeone, Ph.D ${ }^{a, b}$, Kaeli K. Samson, M.S. ${ }^{a}$, Do \\ Young Kim, Ph.D ${ }^{b}$, and Jong M. Rho, M.D. ${ }^{b, c}$ \\ Timothy A. Simeone: timothysimeone@creighton.edu; Kristina A. Simeone: kristinasimeone@creighton.edu; Kaeli K. \\ Samson: kaeli@creighton.edu; Do Young Kim: DoYoung.Kim@chw.edu; Jong M. Rho: \\ jong.rho@albertahealthservices.ca \\ aCreighton University, Department of Pharmacology, Omaha, NE 68174 (U.S.A.) \\ bBarrow Neurological Institute and St. Joseph's Medical Center, Phoenix, AZ 85013 (U.S.A.) \\ 'Alberta Children's Hospital and University of Calgary Faculty of Medicine, Departments of \\ Paediatrics and Clinical Neurosciences, Calgary, Alberta T3B 6A8 (Canada)
}

\section{Abstract}

In human disease, channelopathies involving functional reduction of the delayed rectifier potassium channel a-subunit $\mathrm{K}_{\mathrm{v}} 1.1$ - either by mutation or autoimmune inhibition - result in temporal lobe epilepsy. $\mathrm{K}_{\mathrm{v}} 1.1$ is prominently expressed in the axons of the hippocampal trisynaptic pathway, suggesting its absence will result in widespread effects on normal network oscillatory activity. Here, we performed in vitro extracellular recordings using a multielectrode array to determine the effects of loss of $\mathrm{K}_{\mathrm{v}} 1.1$ on spontaneous sharp waves (SPWs) and high frequency oscillations (HFOs). We found that Kcnal-null hippocampi generate SPWs and ripples (80-200 Hz bandwidth) with a 50\% increased rate of incidence and 50\% longer duration, and that epilepsy-associated pathologic HFOs in the fast ripple bandwidth $(200-600 \mathrm{~Hz})$ are also present. Furthermore, Kcna1-null CA3 has enhanced coupling of excitatory inputs and population spike generation and CA3 principal cells have reduced spike timing reliability. Removing the influence of mossy fiber and perforant path inputs by microdissecting the Kcna1-null CA3 region mostly rescued the oscillatory behavior and improved spike timing. We found that Kcnal-null mossy fibers and medial perforant path axons are hyperexcitable and produce greater pre- and postsynaptic responses with reduced paired-pulse ratios suggesting increased neurotransmitter release at these terminals. These findings were recapitulated in wild-type slices exposed to the $\mathrm{K}_{\mathrm{v}} 1.1$ inhibitor dendrotoxin- $\kappa$. Collectively, these data indicate that loss of $\mathrm{K}_{\mathrm{v}} 1.1$ enhances synaptic release in the $\mathrm{CA} 3$ region, which reduces spike timing precision of individual neurons leading to disorganization of network oscillatory activity and promotes the emergence of fast ripples.

\footnotetext{
(c) 2012 Elsevier Inc. All rights reserved.

Corresponding Author: Jong M. Rho, MD, Division of Pediatric Neurology, Alberta Children's Hospital, 2888 Shaganappi Trail, NW, Calgary, Alberta T3B 6A8 Canada, Tel: 403-955-2635; Fax: 403-955-7649, jong.rho@ albertahealthservices.ca.

Publisher's Disclaimer: This is a PDF file of an unedited manuscript that has been accepted for publication. As a service to our customers we are providing this early version of the manuscript. The manuscript will undergo copyediting, typesetting, and review of the resulting proof before it is published in its final citable form. Please note that during the production process errors may be discovered which could affect the content, and all legal disclaimers that apply to the journal pertain.
} 


\section{Keywords}

High frequency oscillation; epilepsy; potassium channel; sharp wave; hippocampus; $\mathrm{K}_{\mathrm{v}} 1.1$; multielectrode array; ripple; fast ripple; channelopathy

\section{Introduction}

The hippocampus generates a wide array of oscillations such as theta waves $(4-10 \mathrm{~Hz})$, gamma waves $(30-80 \mathrm{~Hz})$ and ripples $(80-200 \mathrm{~Hz})$ which are associated with cognitive processes in vivo. These oscillations are emergent properties of large populations of neurons acting in synchrony (Faingold, 2004). During periods of rest and slow-wave sleep, the CA3 region generates sharp waves (SPWs) and high frequency oscillations (HFOs) in the ripple band that propagate to intra- and extra-hippocampal regions (Buzsaki, 1986). SPW-ripples are hypothesized to participate in memory consolidation via functional alteration of synaptic connectivity and strength (Chrobak et al., 2000; Diba and Buzsaki, 2007; Nakashiba et al., 2009). The cellular and network mechanisms generating SPW-ripples are also related to epileptiform activity as human and rodent epileptic tissue exhibit HFOs in the pathologic fast ripple band (200-600 Hz) (Bragin et al., 1999; Staba et al., 2004; Urrestarazu et al., 2007; Jacobs et al., 2008; Engel et al., 2009). The emergence of pathologic fast ripples in the CA3 region has been associated with several mechanisms, including increased synaptic activity, increased electrical coupling, increased or decreased synchronization and decreased inhibition (Jeffreys et al., 2012). Thus, channelopathies that could affect any one of these mechanisms have the potential of converting network-generated oscillations into pathologic activity. Of particular interest are the $\mathrm{K}_{\mathrm{V}} 1.1$ delayed rectifier voltage-gated potassium channels because of their involvement in many of the above mechanisms and their association with multiple epilepsies.

The $\mathrm{K}_{\mathrm{v}} 1.1 \mathrm{a}$ subunit is localized to axons and axon terminals of the hippocampal trisynaptic circuit and regulates action potential propagation and shape, membrane repolarization, neuronal repetitive firing properties and neurotransmitter release (Geiger and Jonas, 2000; Brew et al., 2003; Shu et al., 2007; Wenzel et al., 2007; Hsiao et al., 2009). Dysfunction or absence of $\mathrm{K}_{\mathrm{v}} 1.1$ is associated with various types of paroxysmal neurological disorders including epilepsy. Specifically, mutations in the homologue gene encoding $\mathrm{K}_{\mathrm{v}} 1.1, K C N A 1$, are associated with partial epilepsy and episodic ataxia (Zuberi et al., 1999; Eunson et al., 2000). Similarly, leucine-rich glioma-inactivated protein-1 (LGI1) gene mutations that result in greater inactivation of $\mathrm{K}_{\mathrm{v}} 1.1$ by the $\mathrm{K}_{\mathrm{v}} \beta 1$ subunit are causative for human autosomal dominant lateral temporal lobe epilepsy (Zhou et al., 2009). Further, sera from cases of human limbic encephalitis, an autoimmune disease associated with epileptic seizures, contain antibodies against $\mathrm{K}_{\mathrm{v}} 1.1$ and/or LGI1, which inhibit channel function (Lalic et al., 2011). In animal models, we and others have demonstrated that targeted deletion of Kcnal in mice results in developmental temporal lobe epilepsy and sudden unexplained death in epilepsy (Smart et al., 1998; Rho et al., 1999; Lopantsev et al., 2003; Wenzel et al., 2007; Fenoglio-Simeone et al., 2009a,b; Glassock et al., 2010). Furthermore, intrahippocampal focal injection of the specific $\mathrm{K}_{\mathrm{v}} 1.1$ antagonist, dendrotoxin- $\mathrm{\kappa}$, induces status epilepticus in rats (Bagetta et al., 1996).

While single cell studies demonstrate that reduction or inhibition of $\mathrm{K}_{\mathrm{v}} 1.1$ function results in significant effects on neurotransmitter release in the hippocampus (Geiger et al., 2000; Zhou et al., 2009; Lalic et al., 2011), the effects on hippocampal network oscillatory behavior remain unknown. Here, we used a planar multi-electrode array to determine the effects of genetic and pharmacologic elimination of $\mathrm{K}_{\mathrm{v}} 1.1$ on SPWs and HFOs. Our results indicate that increased synaptic activity in Kcna1-null CA3 via hyperexcitable mossy fibers and 
medial perforant path axons leads to decreased precision of CA3 principal cell spike timing and the emergence of fast ripples.

\section{Materials and Methods}

Animals

Breeding pairs of heterozygous Kcnal-null mice on a C3HeB/FeJ congenic background were purchased from Jackson Laboratories (Bar Harbor, Maine) and colonies were maintained in the Animal Resource Facilities at Creighton University School of Medicine and the Barrow Neurological Institute. Mice were given food and water ad libitum and kept on a 12-hour light/dark cycle. All procedures involving animals were in accordance with National Institutes of Health guidelines, the EU Directive 2010/63/EU and were approved by the Institutional Animal Care and Use Committees at Creighton University School of Medicine and the Barrow Neurological Institute \& St. Joseph's Medical Center.

\section{Acute slice preparation}

Wild-type and Kcna1-null littermates (P30-P45) were anesthetized with isoflurane, decapitated, and their brains removed and quickly placed into ice cold, oxygenated $\left(95 \% \mathrm{O}_{2} / 5 \% \mathrm{CO}_{2}\right)$ artificial cerebrospinal fluid (aCSF) containing (in $\left.\mathrm{mM}\right): 206$ Sucrose, 2.8 $\mathrm{KCl}, 1 \mathrm{CaCl}_{2}, 1 \mathrm{MgCl}_{2}, 2 \mathrm{MgSO}_{4}, 26 \mathrm{NaHCO}_{3}, 1.25 \mathrm{NaH}_{2} \mathrm{PO}_{4}$, and 10 glucose (pH 7.4). Horizontal sections $(350 \mu \mathrm{M})$ of ventral hippocampal-entorhinal cortex (HEC) were cut on a Leica VT1200 (Leica Microsystems Inc., Bannockburn, IL, USA) and transferred to a holding chamber for $1 \mathrm{hr}$ with warm $\left(32^{\circ} \mathrm{C}\right)$ oxygenated aCSF containing (in $\left.\mathrm{mM}\right): 125$ $\mathrm{NaCl}, 3.0 \mathrm{KCl}, 2.4 \mathrm{CaCl}_{2}, 2.5 \mathrm{MgSO}_{4}, 26 \mathrm{NaHCO}_{3}, 1.25 \mathrm{NaH}_{2} \mathrm{PO}_{4}$, and 10 glucose $(\mathrm{pH}$ 7.4) as we have described (Simeone et al., 2011). All chemicals and drugs were purchased from Sigma-Aldrich (St. Louis, MO, U.S.A.).

\section{Multi-microelectrode recordings}

Procedures were conducted as we have previously described (Simeone et al., 2011). Briefly, a HEC slice was placed over the entire electrode grid of a MED64 probe (see Figure 1A; Alpha Med Systems, Osaka, Japan). A custom probe cap allowed delivery of humidified air $\left(95 \% \mathrm{O}_{2} / 5 \% \mathrm{CO}_{2}\right)$ and perfusion $\left(1 \mathrm{ml} \mathrm{min}^{-1}\right)$ of in-line pre-warmed oxygenated aCSF that resulted in a solution inlet temperature of $\sim 33.2^{\circ} \mathrm{C}$ and an outlet temperature of $\sim 31.6^{\circ} \mathrm{C}$. The bath level was maintained near interface. Spontaneous and evoked extracellular potentials were recorded with either Conductor v3 (Alpha Med Systems) or Mobius v2 (Witwerx Inc., Tustun, CA, USA) software and acquired at a $20 \mathrm{kHz}$ sampling rate with a bandwidth of $0.1 \mathrm{~Hz}-10 \mathrm{kHz}$. Input/output (I/O) curves of presynaptic fiber volleys, postsynaptic field potentials and short-term plasticity were obtained by successive paired stimulations (50 ms inter-stimulus interval) of increasing intensity $(10-180 \mu \mathrm{A})$ separated by $45 \mathrm{sec}$. The mossy fiber (MF)-CA3, medial perforant path (MPP)-dentate granule cell (DG), MPP-CA3, lateral perforant path (LPP)-DG and LPP-CA3 synapses were tested in each slice.

\section{Signal analysis}

Data was imported into Spike2 (v6) software (Cambridge Electronic Design, Cambridge, England) as we have previously described (Simeone et al., 2011). Briefly, to identify DC shifts of the SPWs, raw recordings were subjected to a $50 \mathrm{~Hz}$ low-pass filter $(-3 \mathrm{~dB}$ point $=$ $70 \mathrm{~Hz}$ ) using a Finite Impulse Response (FIR) filter (1319 filter coefficients) provided in the Spike2 software and down-sampled to $2.5 \mathrm{kHz}$. Automated threshold detection of SPWs was set at 3 times the amplitude of the peak-to-peak noise level. SPW frequencies were 
determined by fitting inter-SPW interval histograms with a Gaussian function and calculating the weighted Gaussian mean frequency.

To identify component frequencies of SPWs, raw recordings were down-sampled to $10 \mathrm{kHz}$ and power spectra of the SPWs were produced by applying fast Fourier transforms (Hanning, FFT $=16384$ ) to the raw recordings in $300 \mathrm{~ms}$ windows triggered by the identified SPWs. To verify interpretations of the static power spectra, time-frequency representations of raw recordings were generated with short-time Fourier transform (STFT) analysis in Spike2 software. STFTs were performed with mean detrending, Hanning windowing and FFT size of 2048 points. Once signal frequencies were identified, the raw recordings were filtered with a FIR $100-175 \mathrm{~Hz}$ band pass filter $(-3 \mathrm{~dB}$ points $=80 \mathrm{~Hz}$ and $200 \mathrm{~Hz} ; 1319$ filter coefficients) or a FIR $200-600 \mathrm{~Hz}$ band pass filter $(-3 \mathrm{~dB}$ points $=180$ $\mathrm{Hz}$ and $619 \mathrm{~Hz} ; 1319$ filter coefficients) to visualize ripples and fast ripples, respectively. The root mean square (RMS) of the noise of the filtered recordings was calculated using a 3 ms sliding window. Automated threshold detection of the troughs in each ripple and fast ripple filtered recording was set at 4 times the RMS standard deviation. To determine ripple and fast ripple mean characteristics we performed burst analyses on each recording (Maier et al., 2002, 2003). Identification of ripple bursts required at least three consecutive cycle troughs with inter-trough intervals no greater than $30 \mathrm{~ms}$ in duration $(\geq 33 \mathrm{~Hz})$, whereas fast ripple bursts required at least three consecutive cycle troughs with inter-trough intervals no longer than $6 \mathrm{~ms}(\geq 167 \mathrm{~Hz})$.

Temporal and spatial propagation of SPWs was determined by performing waveform averages (Spike2 software) of events in each of the 64 electrodes. The waveform averages were performed on $300 \mathrm{~ms}$ windows triggered by SPWs identified in an electrode located in the CA3 stratum pyramidale (sp). The temporal difference between electrodes was determined from the time of deviation from baseline of the averaged waveform.

Electrodes in the CA3sp that had visually identifiable multi-unit activity were chosen to examine principal cell and interneuron single unit activity. Recordings were filtered with a FIR $300-3000 \mathrm{~Hz}$ band pass filter. The root mean square (RMS) of the noise of the filtered recordings was calculated using a 3 ms sliding window. Automated threshold detection of the troughs of each unit was set at 4 times the RMS standard deviation. Unit waveform templates were constructed for each recording and used for initial clustering of units into distinct groups. Principal component analysis was used to verify and refine clusters (Spike2 version 6, Cambridge, England). Clusters represented units from single neurons. As previously described, classification of units as belonging to a principal cell or interneuron was based on spike width, asymmetry and autocorrelogram (see Fig. 5 and Table 1) (Csicsvari et al., 1998; Henze et al., 2002; Le Van Quyen et al., 2008). Spike timing jitter was quantified as the mean of the standard deviations of the interspike interval of doublets occurring between SPWs or during SPWs [120 ms window triggered by SPWs identified in the CA3 stratum radiatum (sr)] for each individual principal cell.

For analyzing the stimulation experiments, the post-synaptic field potential slopes (10$90 \%$ ), the fiber volley amplitudes and population spike area were used to quantify responses. Previously, it has been demonstrated that extracellular responses to perforant path stimulation into the CA3 are usually contaminated by polysynaptic inputs via PP-DG-CA3 synapses (Berzhanskaya et al., 1998; Do et al., 2002). To limit this, the slopes (10-90\%) of the first 2 to $3 \mathrm{~ms}$ of the LPP-CA3sr and MPP-CA3sr post-synaptic field potentials were measured (Do et al., 2002). Furthermore, for both genotypes we observed responses that appeared to be a mix MPP and LPP stimulation (e.g., paired-pulse inhibition at low intensities and facilitation at high intensities or vice versa). This has been reported previously (Berzhanskaya et al., 1998); therefore, we only included responses that were 
consistently inhibition or facilitation for all stimulation intensities. I/O relationships of raw and normalized data were fit with a Boltzmann equation to obtain stimulation intensities eliciting 50\% of the maximum response $\left(\mathrm{V}_{50}\right)$ using Prism 6 software (Graphpad Software, Inc., La Jolla, CA, US). Paired-pulse ratios were determined by expressing the second response as a percent of the first response. Mobius v2 software (Witwerx, Inc.) was used for acquisition and analysis of stimulation experiments.

Statistical significance was determined either by an unpaired $t$-test unless otherwise specified using Prism 6 software (Graphpad Software, Inc.) or SigmaStat 3.5 (Systat Software, Inc., Chicago, IL).

\section{Results}

\section{Increased spontaneous SPWs and the emergence of pathologic fast ripples in Kcna1-null hippocampal networks}

Spontaneous SPWs were prevalent and occurred simultaneously in all regions of the hippocampus in slices obtained from wild-type and Kcnal-null mice (Figure 1A). Durations $(50-150 \mathrm{~ms})$ and frequencies $(0.5-4 \mathrm{~Hz})$ of WT SPWs were consistent with previous reports (Maier et al., 2002; Papatheodoropoulos and Kostopoulos, 2002; Maier et al., 2003; Colgin et al., 2004; Wu we al., 2005; Buzsáki, 1996). In both genotypes, there was a 1-2 fold higher SPW incidence in the CA3 region compared to CA1 or dentate gyrus (Figure 1B). Between genotypes, the incidence rate of SPWs was significantly higher by $\sim 43-100 \%$ in all sub-regions throughout the hippocampus of Kcna1-null mice when compared to wild-type controls ( $\mathrm{p}<0.5$; Figure 1B). In addition, the duration of SPWs in CA3sr was $\sim 4 \% \%$ longer in Kcna1-null mice ( $<0.5$; Figure 1B).

Waveform averages were analyzed to determine the spatial-temporal propagation of the SPWs. The earliest initiation times for both wild-type and Kcna 1-null SPWs occurred in the CA3 region (Figure 1C and D). Although propagation time-scales were slice-dependent in both genotypes, the patterns were similar, with SPWs originating in CA3 and spreading to CA1 and DG in under $20 \mathrm{~ms}$. Using a larger multielectrode array $(300 \mu \mathrm{m}$ electrode spacing; Figure 1D), SPWs of both genotypes were found to spread into the entorhinal cortex in under 30 ms. Collectively, these data indicate that: (1) SPWs in both wild-type and Kcna1null slices originate in the CA3 region; (2) 50-60\% of SPWs propagate to other regions; and (3) Kcna1-null SPWs are longer in duration and occur more frequently than in wild-type slices.

To assess the presence of HFOs, power spectral analyses were performed on field recordings of CA3sr of wild-type and Kcna1-null slices. Wild-type power spectra exhibited a sharp peak between 100-200 Hz, indicating the presence of ripples, whereas, Kcna1-null SPWs contained a ripple peak as well as a broad peak between $200-600 \mathrm{~Hz}$, indicating the additional presence of pathologic fast ripples (Figure 2A). Time-frequency analysis revealed spectral disorganization of Kcnal-null HFOs with multiple frequency centers spreading throughout the 100-600 Hz bandwidth (Figure 2B). Burst analyses were performed to quantify ripple and fast ripple characteristics. Compared to wild-type, ripple bursts in Kcna1-null hippocampi were significantly longer in duration, had an increased number of cycles per burst and a lower mean intra-ripple burst frequency (Figure 2C). In Kcna1-null hippocampi, fast ripples were shorter in duration, had a similar number of cycles per burst and the mean intra-HFO burst frequency was three times greater when compared to Kcnalnull ripples (Figure 2C).

Comparisons of the spatial representations of HFOs using power spectral and timefrequency analyses of recordings from all 64 electrodes revealed widespread expression of 
ripples in the hippocampal formation of both genotypes (Figure 2D). Compared to ripples of both genotypes, Kcnal-null fast ripples exhibited greater slice-to-slice variance in the extent of spatial representation, but were generally restricted to the CA3, CA1 and subicular regions. Placement of the HEC slice on a larger array (Figure 2D) revealed prominent ripple activity, but no fast ripples, in the Kcnal-null entorhinal cortex.

\section{Afferent inputs modulate CA3-generated pathologic SPW-HFOs in Kcna1-null hippocampi}

Next, we determined the influence of afferent inputs on SPWs and HFOs in Kcna1-null CA3 by conducting micro-dissection experiments (Figure 3A, B). In agreement with a putative origination site at CA3, SPWs persisted in the CA3-CA1-DG, CA3-CA1 and CA3 minislices (Figure 3C, E, G, respectively) and were absent in isolated entorhinal cortex, isolated DG, and isolated CA1 (Figure 3D, F, H, respectively). SPW duration was $~ 35 \%$ shorter in isolated Kcna1-null CA3-CA1 and CA3 mini-slices when compared to fully intact hippocampal-entorhinal slices (HEC), whereas the rate of incidence remained unchanged (Figure 4A). Mini-slices with the entorhinal cortex, dentate gyrus and/or CA1 removed had significantly shorter ripple and fast ripple durations (Figure 4B). Furthermore, mean intraripple frequencies increased by $\sim 22 \%$ and mean intra-fast ripple frequencies were statistically reduced in the CA3-CA1 and CA3 mini-slices. These changes decreased the fast ripple:ripple frequency ratio from 2.81 in HEC slices to 2.13 in mini-slices. These data suggest that afferent inputs, primarily from the dentate gyrus, significantly influence Kcna1null SPW and HFO frequency and duration; however, are not necessary for SPW-HFO generation.

\section{Afferent Inputs Reduce Spike Timing Reliability of Kcna1-null CA3 Principal Cells}

Spike timing of pairs (doublets) or multiples of action potentials of a single CA3 principal cell is extremely consistent and it enables precise synchronization across small and large networks of CA3 principal cells and underlies ripple oscillations (Foffani et al., 2007; Ibarz et al., 2010). A reduction of spike timing reliability produces out-of-phase firing and promotes the emergence of fast ripples (Foffani et al., 2007; Ibarz et al., 2010). $\mathrm{K}_{\mathrm{v}} 1$ channels, as well as synaptic activity, modulate spike precision (Gittelman and Tempel, 2006; Foffani et al., 2007; Ibarz et al., 2010; Higgs and Spain, 2011; Kuriscak et al., 2012).

We quantified spike timing of extracellular single unit activity in the CA3 principal cell layer in wild-type and Kcnal-null slices to determine whether this mechanism contributes to Kcna1-null fast ripples. In total, we identified 85 principal cells and 80 interneurons. Units were assigned to single cells based on principle component analysis of waveforms and classified as principal cells or interneurons according to spike width, asymmetry and autocorrelogram (Figure 5A and Table 1) (Csicsvari et al., 1998; Henze et al., 2002; Le Van Quyen et al., 2008). The autocorrelogram provides information on the firing patterns of the cell; thus, principal cells that have a tendency to burst usually have a peak between $2-8 \mathrm{~ms}$ with a fast decay, whereas interneurons have a less pronounced peak between 7-40 ms with a broad decay representing a more consistent firing in between short periods of bursting (Figure 5A). Bursting for both cell types frequently occurred during SPW-HFO events. These dynamics gave the interneurons a larger mean autocorrelogram when quantified (Table 1). Cell type characteristics did not differ between genotypes with the exception that Kcna1-null interneuron firing frequency was twice that compared to wild-type ( $\mathrm{p}<0.001)$. We assessed Kcnal-null CA3 mini-slices to determine whether differences are due to afferent inputs to CA3 or due to the lack of $\mathrm{K}_{\mathrm{v}} 1.1$ in CA3 principal cells (Gittelman and Tempel, 2006; Higgs and Spain, 2011). Isolating the Kcnal-null CA3 significantly reduced the interneuron firing frequency $(\mathrm{p}=0.001)$ to near wild-type levels, whereas Kcnal-null principal cell firing increased to a rate significantly higher than wild-type $(\mathrm{p}<0.05)$. The 
means of the autocorrelograms were greater for both cell types in the Kcnal-null CA3 minislices. This may reflect increased regular firing between bursts.

To determine spike timing reliability of the principal cells, we quantified the interspike interval (ISI) and jitter (mean of standard deviations of ISIs for individual neurons) of doublet spikes occurring in between SPWs. We restricted our analysis to doublets firing in the $130-180 \mathrm{~Hz}$ range to ensure comparison of similar firing patterns and increase the sensitivity of detecting differences in jitter (Foffani et al., 2007). The average ISI was about $6.5 \mathrm{~ms}$ or $154 \mathrm{~Hz}$ (i.e., close to the wild-type ripple frequency) in wild-type, Kcna1-null and Kcna1-null mini-slices (Figure 5B, C). Kcna1-null CA3 principal cell spike jitter was significantly higher than wild-type $(\mathrm{p}<0.05)$. Transecting the afferent inputs and isolating CA3 reduced the doublet jitter (Figure 5B, C).

Next we analyzed the ISIs and jitter of the principal cells during SPWs. We found that mean population ISIs during SPWs were below the ripple frequency for both genotypes (Figure 5D). This concurs with previous reports of CA3 principal cells displaying diverse firing patterns during SPWs, i.e. some fire continuously, some fire during specific phases and some are silenced by the SPW (Foffani et al. 2007; Spampanato and Mody, 2007; Ibarz et al., 2010). Furthermore, individual neurons had variable firing patterns across SPWs that gave rise to rather large jitter (Figure 5D). This is to be expected because CA3 principal cells tend to have high firing rates at the beginning of HFOs that continuously slow throughout the HFO (Spampanato and Mody, 2007). Kcna1-null principal cells displayed larger SPW-spike ISIs and jitter compared to wild-type (Figure 5D). These were returned to near wild-type levels in the Kcnal-null CA3 mini-slice. Collectively, these results suggest that afferent inputs to Kcna1-null CA3 increase the jitter of Kcna1-null principal cells, which may contribute to the emergence of fast ripples.

\section{Kcna1-null Granule Cell Mossy Fibers and Medial Perforant Path Axons are Hyperexcitable with Decreased Paired Pulse Ratios}

Removal of the entorhinal cortex and dentate gyrus resulted in significant effects on Kcna1null SPW and HFO characteristics supporting the possibility that there may be a dysregulation of synaptic activity of the primary inputs into the CA3 region (Figure 4). To investigate synaptic properties that could underlie the altered network activity, we performed I/O experiments with paired stimulations (50 ms interstimulus interval) and determined pre-synaptic fiber volley amplitudes, post-synaptic field potential slopes and paired-pulse ratios. An electrode in the hilar region of the dentate gyrus stimulated mossy fibers of granule cells and responses were examined in the CA3 stratum lucidum (sl) where the mossy fibers synapse onto principal cell dendrites (Figure 6A, B). Kcna1-null mossy fiber presynaptic volleys were $227 \%$ larger than wild-type and required $30 \%$ lower stimulation intensities to elicit a half-maximal response (Figure 6C; Table 2). Similarly, the Kcna1-null field potential slopes were 169\% larger and required 24\% lower stimulation intensities to elicit a half-maximal response compared to wild-type (Figure 6D; Table 2). Linear fits of normalized field potential slopes as a function of normalized fiber volley amplitudes revealed no difference in pre- and post-synaptic coupling (regression slopes: $1.004 \pm 0.07$ WT vs. $0.985 \pm 0.07$ Kcnal-null; $\mathrm{p}=0.849$ ) suggesting that the increased recruitment of KO fibers were responsible for the increased fEPSPs.

Another factor affecting spontaneous and evoked neurotransmission is release probability. Mossy fiber terminals have low neurotransmitter release probabilities resulting in relatively large paired-pulse ratios (i.e., facilitation) of the second postsynaptic response. Because field potentials may be contaminated by unintended offtarget fiber stimulation or polysynaptic feed-forward excitation or inhibition (especially at higher stimulation intensities), we quantified the paired-pulse ratio over a wide-range of stimulation intensities $(10-180 \mu \mathrm{A})$. 
Compared to wild-type, Kcna1-null mossy fiber-CA3sl paired pulse facilitation was 23 $46 \%$ lower (Fig 6). Mossy fiber stimulation was confirmed by application of the group II metabotropic glutamate receptor agonist, DCG-IV, which inhibits mossy fiber mediated responses (data not shown). Collectively, these results suggest that Kcna1-null mossy fibers are hyperexcitable and release more neurotransmitter, which may be due to increased axonal recruitment and increased release probabilities.

Additional inputs into CA3 are via perforant path monosynaptic and disynaptic connections. The excitatory axons from the entorhinal cortex travel in the perforant fiber paths (MPP and LPP) and form synapses with dentate granule cell dendrites in the molecular layers of the dentate gyrus and, to a lesser degree, dendrites of CA3 principal cells in the CA3sr (Figure 7A, B). It is important to note that: 1) MPP axons terminate in the inner/middle molecular layers, whereas LPP axons terminate in the outer/middle molecular layers, 2) MPP exhibits paired pulse inhibition and LPP has paired pulse facilitation and 3) MPP axons and terminals prominently express $\mathrm{K}_{\mathrm{v}} 1.1$, whereas LPP lack $\mathrm{K}_{\mathrm{v}} 1.1$ (Wenzel et al., 2007). Accordingly, responses to LPP stimulations did not differ between genotypes (Table 2). However, MPP exhibited differences similar to those observed with mossy fiber stimulation. Kcna1-null MPP-DG fiber volleys were 179\% larger than wild-type and required 29\% lower stimulation intensities to elicit a half-maximal response (Figure 7C; Table 2). The Kcna1null MPP-DG field potential slopes were 230\% larger and required 23\% lower stimulation intensities to elicit a half-maximal response compared to wild-type (Figure 7D; Table 2). Fiber volley-field potential coupling was not different (1.057 \pm 0.06 WT vs. $0.974 \pm 0.05$ Kcna1-null; p=0.317) (Figure 7E). Compared to wild type, Kcna1-null MPP-DG had 176 $308 \%$ greater paired pulse inhibition over the range of stimulation intensities with the greatest inhibition occurring with the smaller responses (Figure 7F). MPP also directly synapses onto distal CA3 dendrites; therefore, we analyzed electrodes in the CA3sr. As indicated in Table 2, we found the MPP-CA3sr responses were comparable to MPP-DG for both genotypes. Kcna1-null MPP-CA3sr were hyperexcitable, had larger field potentials and greater paired pulse inhibition compared to wild-type (Table 2). Collectively, these data suggest increased synaptic activity in Kcna1-null CA3 via direct synapses from hyperexcitable mossy fibers and MPP and via a disynaptic MPP-DG-CA3 route.

\section{Kcna1-null CA3 has Enhanced Coupling between fEPSPs and Population Spikes}

Changes in synaptic activity and/or $\mathrm{K}_{\mathrm{v}} 1.1$ expression are predicted to affect the excitability of neurons, and thus their spike timing (McKay et al., 2005; Kuriscak et al., 2012). To determine if Kcna1-null CA3 neurons are hyperexcitable, we assessed the coupling of excitatory inputs and firing efficiency. We analyzed electrodes in CA3sp upon mossy fiber stimulation (Figure 8A). Compared to wild-type, Kcnal-null fEPSP slopes (max. response $298.4 \pm 66.4 \mu \mathrm{V} \mathrm{ms}^{-1} ; \mathrm{n}=5$ slices) were significantly smaller than wild-type (max. response: $618.4 \pm 79.4 \mu \mathrm{V} \mathrm{ms}^{-1} ; \mathrm{n}=7$ slices; $\mathrm{p}<0.05$; Figure $8 \mathrm{~B}$ ), whereas the population spikes did not change $(\mathrm{p}=0.996)$. fEPSP-population spike $(\mathrm{E}-\mathrm{S})$ coupling ratios were obtained from the linear regression slopes of the E-S plots. Due to the difference in fEPSP slopes, the Kcna1-null E-S coupling was $227 \%$ greater than wild-type $(2.08 \pm 0.12 \mathrm{WT}$ vs. $4.73 \pm 0.18$ Kcnal-null; p <0.001) (Figure 8C). These data indicate that Kcna1-null CA3 neurons have increased excitability (i.e., firing occurred with less excitatory input).

\section{Pharmacological Inhibition of $\mathrm{K}_{\mathrm{v}} 1.1$ Recapitulates the Kcna1-null Oscillatory Phenotype}

To determine whether the morphological changes described in the Kcan1-null hippocampus (Wenzel et al., 2007) or the lack of $\mathrm{K}_{\mathrm{v}} 1.1$ subunits contribute to alterations in network oscillatory behavior, we perfused wild-type slices with dendrotoxin- $\kappa$ (DTX-k), a selective $\mathrm{K}_{\mathrm{v}} 1.1$ inhibitor (Akhatar et al., 2002). Application of $100 \mathrm{nM}$ DTX-k mimicked the genetic deletion of $\mathrm{K}_{\mathrm{v}} 1.1$ (Figure 9). Namely, DTX-k increased in SPW frequency (47\%) and 
duration (25\%), increased ripple durations (35\%), reduced intra-ripple frequencies and produced the emergence of fast ripples and further spectral disorganization (Figure 9B, C). Prolonged exposure to DTX-k ( $>40 \mathrm{~min}$ ) resulted in regular, large amplitude interictal-like events (inter-ILEs) that were associated with longer duration ripples and fast ripples (Figure 9C). The fast ripple:ripple frequency ratio increased from 2.43 (during early DTX-k) to 2.58 during inter-ILEs. These changes were associated with increased CA3 principal cell spike timing jitter during doublets and SPWs (Figure 9D; Table 1). Multi-units occurred at extremely high rates during inter-ILEs and could not be distinguished with certainty; therefore, we did not analyze single units. Additionally, DTX-k reduced the paired pulse ratios at the MF-CA3 and MPP-DG synapses during early-stage DTX-k perfusion (Figure 9E, Table 2). However, DTX-k did not change either mossy fiber or MPP excitability since $\mathrm{V}_{50}$ 's and maximum responses did not significantly differ from baseline ( $\mathrm{n}=7$ slices; data not shown). Stimulation during prolonged DTX-k exposure evoked epileptiform activity resulting in indiscernible responses that did not enable accurate measurement of field potentials and paired pulse ratios (data not shown). These results suggest that loss of $\mathrm{K}_{\mathrm{v}} 1.1$ function is sufficient to convert SPWs into pathologic SPWs, ripples into pathologic ripples, reduce spike timing reliability and promote the emergence of fast ripples.

\section{Discussion}

Hippocampal sharp waves and high frequency oscillations are intimately involved in cognitive processes and epileptiform activity. Synaptic activity (excitatory and inhibitory), gap junctions and ephaptic interactions are thought to modulate the CA3 generator of SPWs and HFOs and participate in the emergence of pathologic fast ripples (Jeffreys et al., 2012). The present study is the first to explore the effect of a $\mathrm{K}_{\mathrm{v}} 1.1$ channelopathy on network oscillatory behavior. We demonstrate that the loss of $\mathrm{K}_{\mathrm{v}} 1.1$ either by genetic or pharmacologic manipulation alters in vitro hippocampal network oscillatory patterns and promotes the emergence of pathologic fast ripples. Collectively, our data suggest that these effects stem from significant functional alterations of dentate gyrus and medial perforant path inputs into CA3 and increased synaptic activity. We found: (1) Hippocampal networks lacking $\mathrm{K}_{\mathrm{v}} 1.1$ generate SPWs and HFOs at a higher incidence rate, are longer in duration, have lower intra-ripple frequencies, spontaneously generate pathologic fast ripples and have reduced spike timing precision of $\mathrm{CA} 3$ principal cells. (2) Isolation of $\mathrm{CA} 3$ from dentate gyrus and entorhinal inputs reduce the duration of SPWs and HFOs, increase the intra-ripple frequencies, lowers the intra-fast ripple frequency and improves spike timing reliability. (3) The mossy fiber and MPP axons are hyperexcitable and have reduced synaptic paired pulse ratios in Kcnal-null hippocampi (i.e., indicating increased release probabilities). (4) Pharmacological inhibition of $\mathrm{K}_{\mathrm{v}} 1.1$ in wild-type slices recapitulates the oscillatory phenotype of the Kcna1-null hippocampal network.

Previously, we and others have shown that mice lacking the $\mathrm{K}_{\mathrm{v}} 1.1 \mathrm{a}$-subunit naturally develop epilepsy of limbic origin and have more than half a dozen spontaneous seizures per day (Smart et al., 1998; Wenzel et al., 2007; Fenoglio-Simeone et al., 2009a,b). The $\mathrm{K}_{\mathrm{v}} 1.1$ a-subunit is localized to juxtaparanodal regions of myelinated and unmyelinated axons and synaptic terminals. It is highly expressed throughout the hippocampal network: specifically, on the afferent projections of the medial perforant path, dentate granule cell mossy fibers, Schaffer collaterals of CA3 pyramidal cells, and in the interneurons of the dentate-hilar regions and CA1 pyramidal layer (Zucker et al., 2002; Wenzel et al., 2007). Based on its location and biophysical properties, lack of $\mathrm{K}_{\mathrm{v}} 1.1$ a-subunit could increase the probability of synaptic activity by multiple mechanisms, including promoting axonal resting membrane depolarization, action potential broadening and slowing repolarization (Geiger et al., 2000; Brew et al., 2003; Shu et al., 2007). Indeed, single cell studies demonstrate that experimental reduction or pharmacological inhibition of $\mathrm{K}_{\mathrm{v}} 1.1$ function results in significant increase of 
neurotransmitter release in the hippocampus (Geiger et al., 2000; Zhou et al., 2009; Lalic et al., 2011). Here, we found that Kcnal-null synapses involving mossy fibers and the MPP have reduced paired-pulse ratios. These may reflect increased release probabilities (Zucker et al., 2002) and further support and expand on the previous in vitro studies that have utilized wild-type hippocampal tissue. Release probability of mossy fiber-to-CA3 synapses is increased following treatment with sera containing antibodies against $\mathrm{K}_{\mathrm{v}} 1.1$ isolated from human limbic encephalitis patients (Lalic et al., 2011). A second study reported increased neurotransmitter release probabilities at the MPP-to-granule cell synapse in hippocampal slices expressing a mutated $L G I 1$ gene, which promotes the inactivation of $\mathrm{K}_{\mathrm{v}} 1.1$ and is associated with human autosomal dominant lateral temporal lobe epilepsy (Zhou et al., 2009). Neurotransmitter release is also markedly enhanced in cultured hippocampal neurons that over-express human $\mathrm{K}_{\mathrm{v}} 1.1$ subunits containing mutations that are associated with episodic ataxia and epilepsy (Heeroma et al., 2009). In addition, we determined that the specific $\mathrm{K}_{\mathrm{v}} 1.1$ antagonist, dendrotoxin- $\kappa$, reduced paired-pulse ratios at both mossy fiberCA3 and MPP-mossy fiber synapses in wild-type hippocampal slices, which is supported by similar studies in which a-dendrotoxin, a nonspecific $\mathrm{K}_{\mathrm{v}} 1.1,1.2$ and 1.6 antagonist, resulted in increased release probabilities (Zhou et al., 2009; Lalic et al., 2011). Our studies using tissue slices isolated from Kcnal-null epileptic mice further support the notion that functional reduction of $\mathrm{K}_{\mathrm{v}} 1.1$ is associated with increased neurotransmitter release within the hippocampal network and epilepsy. Importantly, decreases in paired-pulse ratios of mossy fiber and MPP synapses occur in multiple animal models of epilepsy, raising the possibility that increases in neurotransmitter release probabilities, specifically at these synapses, may be a general phenomena and contributing factor in epilepsy (Clusmann et al., 1992; Goussakov et al., 2000; Sloviter, 1992; Buhl et al., 1996; Buckmaster and Dudek, 1997; Wu and Leung, 2001).

Increased glutamate release by MPP axons onto dentate granule cells and by granule cell mossy fibers onto dendrites in the CA3 region would suggest a substantial increase in feedforward activity in the CA3 region. Indeed, we found a significantly higher incidence in SPW generation and longer durations of both sharp waves and HFOs. SPWs are spontaneous field potentials arising from emerging, synchronous bursts of large populations of recurrently connected CA3 pyramidal cells (Csicsvari et al., 2000). CA3 HFOs are population spikes of a sub-network of pyramidal cells that synchronously fire action potentials and are part of the population responsible for the SPWs (Foffani et al 2007; Ibarz et al., 2010). CA3 pyramidal neurons are prone to generate bursts of action potentials via intrinsic mechanisms that are highly sensitive to minor synaptic provocations, and synchronize by means of recurrent collaterals and gap junctions (Prince and Connors, 1986; Traub et al., 2002). SPW and HFO duration reflects the time-course of transmembrane currents of the underlying population of neurons and is therefore also sensitive to small changes in synaptic events (Maier et al., 2003; Buzsáki, 1996; Behrens et al., 2005). Inhibition of $\mathrm{K}_{\mathrm{v}} 1.1$ reduces synaptic transmission failure rates (i.e. increases the probability of release), which may lengthen the time of transmitter in the synaptic cleft either due to increased concentration of transmitter or increased overlap of release events (Geiger et al., 2000; Zhou et al., 2009; Lalic et al., 2011). We found that removal of the entorhinal cortex from Kcna1-null hippocampal slices reduced ripple and fast ripple durations presumably by decreasing activity of perforant path axons. Removing the dentate gyrus had a more pronounced effect by reducing SPW durations and further reduced ripple durations to near wild-type levels. Neither micro-dissections affected the SPW incidence. These results suggest four conclusions. First, Kcna1-null MPP axons and mossy fibers are hyperactive which is concordant with our fiber volley and paired pulse data. Second, the dentate gyrus exerts significant modulatory control over the CA3 oscillatory generator in Kcna1-null slices. This finding concurs with a previous report on hippocampal $\beta / \gamma$ oscillations (Trevino et al., 2007) and extends this conclusion into the high frequency range. Third, the large 
neuronal networks underlying SPWs require significant increases in synaptic activity to lengthen durations as it is affected only when the numerous dentate gyrus inputs are removed. Because the sub-networks responsible for generating HFOs are part of the larger SPW-population it is not surprising that HFO duration is also affected by removal of the dentate. What is surprising is that transection of the perforant path has a significant effect on HFO durations. Perhaps increased polysynaptic activity via mossy fibers or coincident activation of CA3 by monosynaptic perforant path axons and mossy fibers lengthens the time that small networks can remain synchronized. This remains to be studied. Finally, SPW incidence may either be highly sensitive to the level of local synaptic activity and affected by increased constitutive release of the remaining mossy fiber terminals in the Kcnal-null CA3 mini-slice, as found in slices from wild-type rats (Rex et al., 2009), and/or may be due to an increase in the intrinsic bursting properties of Kcnal-null CA3 principal cells (Smart et al., 1998; Lopantsev et al., 2003; Dzhala and Staley, 2004).

The dentate gyrus inputs also modulate the oscillatory range of HFOs as isolation of the Kcna1-null CA3 increases the mean intra-ripple frequencies and decreases the mean intrafast ripple frequencies. Fast ripples may emerge from a degeneration of the synchronization of firing pyramidal cells underlying ripples via increased jitter of spike timing (Foffani et al., 2007; Ibarz et al., 2010). Using elegant in silico modeling experiments, Ibarz and colleagues (2010) predicted that increases in either synaptic strength or synaptic noise would promote out-of-phase firing of the principal cells, formation of sub-clusters, introduction of additional population spikes and ultimately spectral disorganization and the emergence of fast ripples. These events are very similar to those observed for Kcnal-null slices or wildtype slices exposed to DTX-k. Specifically, we found that loss of $\mathrm{K}_{\mathrm{v}} 1.1$ results in CA3 neurons having enhanced $\mathrm{E}-\mathrm{S}$ coupling reflecting reduced firing thresholds to smaller inputs (Figure 8), increased principal cell spike timing jitter (Figure 5 and Figure 9), increased spike ISIs during SPWs (Figure 5 and Figure 9) and spectral disorganization as depicted in time frequency analyses (Figure 2 and Figure 9). Alterations of mossy fibers and MPP axons suggest increases in synaptic strength and/or noise (Figs. 6, 7,9 and Table 2), but it is also possible that elimination of $\mathrm{K}_{\mathrm{v}} 1.1$ in CA3 principal cells contributes to the above cellular changes and fast ripple emergence. Although $\mathrm{K}_{\mathrm{v}} 1.1$ does not affect somatic membrane potential, input resistance or action potential waveforms (Smart et al., 1998; Lopantsev et al., 2003; Shu et al., 2007), pharmacologic or genetic deletion of $\mathrm{K}_{\mathrm{v}} 1.1$ does reduce the dynamic control of action potential threshold voltages allowing smaller and/or longer duration inputs to initiate spikes with the same efficiency as short and/or large inputs, thereby increasing the temporal window of spikes and jitter (Gittelman and Tempel, 2006; Higgs and Spain, 2011). This could decrease spike coherence with high frequency inputs by increasing the lower frequency signal modulation of spike timing (McKay et al., 2005; Higgs and Spain, 2011). Accordingly, it would be predicted that the longer duration Kcna1null and DTX-k SPWs and presumed increased asynchronous synaptic activity would elicit a wider range of firing patterns resulting in increased jitter and mean ISIs as we observed for single units of CA3 principal cells. Thus, it is likely that fast ripples are due to not only increased synaptic activity in CA3, but also an increased sensitivity of the principal cells to said synaptic activity. This may explain the continued presence of fast ripples in the isolated CA3 mini-slices.

At this time the nature of the synaptic activity is unknown, but is suspected to be complex and dynamic involving both excitation and inhibition. Mossy fibers synapse onto both CA3 pyramidal cells and interneurons and release not only glutamate, but also GABA and a variety of neuropeptides (Jaffe and Guiterrez, 2007). Interneurons are known to be involved in CA3 HFOs (Spampanato and Mody, 2007) and we observed an increase in interneuron firing frequencies (Table 2) suggesting a possible role in fast ripple generation in this model of epilepsy. Furthermore, the GABA content of mossy fiber terminals increases in multiple 
models of epilepsy (Jaffe and Guiterrez, 2007) and, as in human temporal lobe epilepsy, Kcna1-null hippocampi sprout additional mossy fibers (Wenzel et al., 2007). Therefore, we suspect that an increase in mossy fiber neurotransmitter release results in both increased excitatory and inhibitory noise in Kcna1-null CA3.

The oscillatory phenotype of the Kcna1-null hippocampi was recapitulated by pharmacological inhibition of $\mathrm{K}_{\mathrm{v}} 1.1$ channels with DTX-k application to slices from wildtype mice. This suggests that the restructuring observed in Kcnal-null hippocampi (Wenzel et a., 2007) may not be necessary for the emergence of fast ripples as has been suggested for other models of epilepsy (Engel et al., 2009). Previous studies indicate whilst cytoarchitectural changes certainly have the potential to contribute to pathologic HFO emergence, channelopathies that affect synaptic noise and integration are sufficient (Bragin et al., 2002; Bernard et al., 2004; Simeone et al., 2005; Foffani et al., 2007; Richichi et al., 2008; Marcelin et al., 2009). The current study provides a prime example of the consequences of a channelopathy on emergent properties of neural networks. An interesting difference between genetic and pharmacologic manipulations was the development of interictal-like events after prolonged inhibition of $\mathrm{K}_{\mathrm{v}} 1.1$. This may have important implications for autoimmune diseases that target $\mathrm{K}_{\mathrm{v}} 1.1$ (Lalic et al., 2011). Similar to the subunit-dependence of pharmacological modulation of other ion channels, sensitivity to DTX-k is directly proportional to the number of $\mathrm{K}_{\mathrm{v}} 1.1$ subunits present in a heteromeric potassium channel (Akhatar et al., 2002; Simeone et al., 2006, 2011b, 2011c). Thus, the time-dependent emergence of inter-ictal like events is likely due to the progressive block of less sensitive heteromeric subunit combinations. This finding also suggests the intriguing possibility that an unknown homeostatic change occurs in the Kcnal-null hippocampus that is responsible for preventing the spontaneous generation of interictal-like spikes in vitro. Recent evidence would argue against up-regulation of other delayed rectifier potassium channel subunits as the homeostatic mechanism (Wenzel et al., 2007; Menegola et al., 2012). Discovering this "breaking" mechanism will illuminate the manner by which a hyperexcitable hippocampus transitions to seizures and explain the spontaneous nature of in vivo seizures in this model of temporal lobe epilepsy.

In conclusion, our results indicate that hippocampal networks that lack $\mathrm{K}_{\mathrm{v}} 1.1$ have increased synaptic release by mossy fibers and MPP which plays a significant role in the development of pathologic SPWs, ripples and the emergence of fast ripples. The present study is immediately relevant to several human temporal lobe epilepsies involving both mutations of and autoantibodies for $\mathrm{K}_{\mathrm{v}} 1.1$ or LGI1 (Zuberi et al., 1999; Eunson et al., 2000; Zhou et al., 2009; Lalic et al., 2011); but, may also be generalized to other temporal lobe epilepsies as decreased paired-pulse ratios of mossy fiber and medial perforant path synapses are common features of other animal models of epilepsy (Clusmann et al., 1992; Goussakov et al., 2000; Sloviter et al., 1992; Buhl et al., 1996; Buckmaster and Dudek, 1997; Wu and Leung, 2001). Given that the cellular and network substrates necessary to generate SPWripples are thought to be the same as those required for epileptiform discharges (Engel et al., 2009; Dichter, 2009), continued investigation into the network, cellular and molecular mechanisms leading to pathologic SPW-HFOs will shed light on the mechanism underlying transitions to interictal and ictal events.

\section{Acknowledgments}

The authors thank Stephanie Matthews and Heather Milligan for their technical assistance. This work was supported by the Health Future Foundation (TAS), NIH grants NS044846 (JMR), NS070261 (JMR) and NS072179-02 (KAS) and the Barrow Neurological Foundation (JMR). The project described was also supported by the National Center for Research Resources grant G20RR024001. The content is solely the responsibility of the authors and does not necessarily represent the official views of the National Center for Research Resources or the National Institutes of Health. 


\section{References}

Akhtar S, Shamotienko O, Papakosta M, Ali F, Dolly JO. Characteristics of brain Kv1 channels tailored to mimic native counterparts by tandem linkage of alpha subunits: implications for $\mathrm{K}+$ channelopathies. J Biol Chem. 2002; 277:16376-16382. [PubMed: 11859070]

Bagetta G, Iannone M, Palma E, Nisticò G, Dolly JO. N-methyl-D-aspartate and non-N-methyl-Daspartate receptors mediate seizures and CA1 hippocampal damage induced by dendrotoxin-K in rats. Neuroscience. 1996; 71:613-624. [PubMed: 8867035]

Behrens CJ, van den Boom LP, de Hoz L, et al. Induction of sharp wave-ripple complexes in vitro and reorganization of hippocampal networks. Nature Neurosci. 2005; 8:1560-1567. [PubMed: 16222227]

Bernard C, Anderson A, Becker A, Poolos NP, Beck H, Johnston D. Acquired dendritic channelopathy in temporal lobe epilepsy. Science. 2004; 305:532-535. [PubMed: 15273397]

Berzhanskaya J, Urban NN, Barrionuevo G. Electrophysiological and pharmacological characterization of the direct perforan path input to hippocampal CA3. J Neurophysiol. 1998; 79:2111-2118. [PubMed: 9535972]

Bragin A, Engel J Jr, Wilson CL, Fried I, Mathern GW. Hippocampal and entorhinal cortex highfrequency oscillations $(100--500 \mathrm{~Hz})$ in human epileptic brain and in kainic acid--treated rats with chronic seizures. Epilepsia. 1999; 40:127-137. [PubMed: 9952257]

Bragin A, Mody I, Wilson CL, Engel J Jr. Local generation of fast ripples in epileptic brain. J Neurosci. 2002; 22:2012-2021. [PubMed: 11880532]

Bragin A, Wilson CL, Engel J Jr. Voltage depth profiles of high-frequency oscillations after kainic acid-induced status epilepticus. Epilepsia. 2007; 48:S35-S40.

Brew HM, Hallows JL, Tempel BL. Hyperexcitability and reduced low threshold potassium currents in auditory neurons of mice lacking the channel subunit $\mathrm{K}_{\mathrm{V}} 1.1$. J Physiol. 2003; 548:1-20. [PubMed: 12611922]

Buckmaster PS, Dudek FE. Network properties of the dentate gyrus in epileptic rats with hilar neuron loss and granule cell axon reorganization. J Neurophysiol. 1997; 77:2685-2696. [PubMed: 9163384]

Buhl EH, Otis TS, Mody I. Zinc-induced collapse of augmented inhibition by GABA in a temporal lobe epilepsy model. Science. 1996; 271:369-373. [PubMed: 8553076]

Buzsáki G. Hippocampal sharp waves: Their origin and significance. Brain Research. 1996; 398:242252. [PubMed: 3026567]

Chrobak JJ, Lörincz A, Buzsáki G. Physiological patterns in the hippocampo-entorhinal cortex system. Hippocampus. 2000; 10:457-465. [PubMed: 10985285]

Clusmann H, Stabel J, Stephens DN, Heinemann U. Alterations in medial perforant path and mossy fiber induced field potentials in amygdala and beta-carboline (FG 7142) kindled rats. Neurosci Lett. 1992; 146:65-68. [PubMed: 1475051]

Colgin LL, Kubota D, Jia Y, et al. Long-term potentiation is impaired in rat hippocampal slices that produce spontaneous sharp waves. J Physiol. 2004; 558:953-961. [PubMed: 15194734]

Csicsvari J, Hirase H, Czurko A, Buzsáki G. Reliability and state dependence of pyramidal cellinterneuron synapses in the hippocampus: an ensemble approach in the behaving rat. Neuron. 1998; 21:179-189. [PubMed: 9697862]

Csicsvari J, Hirase H, Mamiya A, Buzsáki G. Ensemble patterns of hippocampal CA3-CA1 neurons during sharp wave-associated population events. Neuron. 2000; 28:585-594. [PubMed: 11144366]

Diba K, Buzsáki G. Forward and reverse hippocampal place-cell sequences during ripples. Nat Neurosci. 2007; 10:1241-1242. [PubMed: 17828259]

Dichter MA. Emerging concepts in the pathogenesis of epilepsy and epileptogenesis. Arch Neurol. 2009; 66:443-447. [PubMed: 19364928]

Do VH, Martinez CO, Martinez JL, Derrick BE. Long-term potentiation in direct perforant path projections to the hippocampal CA3 region in vivo. J Neurophysiol. 2002; 87:669-678. [PubMed: 11826036] 
Dzhala VI, Staley KJ. Mechanisms of fast ripples in the hippocampus. J Neurosci. 2004; 24:88968906. [PubMed: 15470156]

Engel J Jr, Bragin A, Staba R, Mody I. High-frequency oscillations: What is normal and what is not? Epilepsia. 2009; 50:598-604. [PubMed: 19055491]

Eunson LH, Rea R, Zuberi SM, et al. Clinical, genetic, and expression studies of mutations in the potassium channel gene Kcna1 reveal new phenotypic variability. Ann Neurol. 2000; 48:647-656. [PubMed: 11026449]

Faingold CL. Emergent properties of CNS neuronal networks as targets for pharmacology: application to anticonvulsant drug action. Prog Neurobiol. 2004; 72:55-85. [PubMed: 15019176]

Fenoglio-Simeone K, Mazarati A, Sefidvash-Hockley S, Shin D, Wilke J, Milligan H, Sankar R, Rho JM, Maganti R. Anticonvulsant effects of the selective melatonin receptor agonist ramelteon. Epilepsy Behav. 2009a; 16:52-57. [PubMed: 19682955]

Fenoglio-Simeone KA, Wilke JC, Milligan HL, Allen CN, Rho JM, Maganti RK. Ketogenic diet treatment abolishes seizure periodicity and improves diurnal rhythmicity in epileptic Kcna1-null mice. Epilepsia. 2009b; 50:2027-2034. [PubMed: 19490051]

Foffani G, Uzcategui YG, Gal B, Menendez de la Prida L. Reduced spike-timing reliability correlates with the emergence of fast ripples in the rat epileptic hippocampus. Neuron. 2007; 55:930-941. [PubMed: 17880896]

Geiger JR, Jonas P. Dynamic control of presynaptic $\mathrm{Ca}(2+)$ inflow by fast-inactivating $\mathrm{K}(+)$ channels in hippocampal mossy fiber boutons. Neuron. 2000; 28:927-939. [PubMed: 11163277]

Gittelman JX, Tempel BL. Kv1.1-containing channels are critical for temporal precision during spike initiation. J Neurophysiol. 2006; 96:1203-1214. [PubMed: 16672305]

Glasscock E, Yoo JW, Chen TT, et al. $\mathrm{K}_{\mathrm{V}} 1.1$ potassium channel deficiency reveals brain-driven cardiac dysfunction as a candidate mechanism for sudden unexplained death in epilepsy. $\mathbf{J}$ Neurosci. 2010; 30:5167-5175. [PubMed: 20392939]

Goussakov IV, Fink K, Elger CE, Beck H. Metaplasticity of Mossy Fiber Synaptic Transmission Involves Altered Release Probability. J Neurosci. 2000; 20:3434-3441. [PubMed: 10777806]

Hsiao CF, Kaur G, Vong A, Bawa H, Chandler SH. Participation of Kv1 channels in control of membrane excitability and burst generation in mesencephalic V neurons. J Neurophysiol. 2009; 101:1407-1418. [PubMed: 19144742]

Heeroma JH, Henneberger C, Rajakulendran S, et al. Episodic ataxia type 1 mutations differentially affect neuronal excitability and transmitter release. Dis Model Mech. 2009; 2:612-619. [PubMed: 19779067]

Henze DA, Wittner L, Buzsáki G. Single granule cells reliably discharge targets in the hippocampal CA3 network in vivo. Nat Neurosci. 2002; 5:790-795. [PubMed: 12118256]

Higgs MH, Spain WJ. Kv1 channels control spike threshold dynamics and spike timing in cortical pyramidal neurones. J Physiol. 2011; 589:5125-5142. [PubMed: 21911608]

Ibarz JM, Foffani G, Cid E, Inostroza M, Menendez de la Prida L. Emergent dynamics of fast ripples in the epileptic hippocampus. J Neurosci. 2010; 30:16249-16261. [PubMed: 21123571]

Jacobs J, LeVan P, Chander R, Hall J, Dubeau F, Gotman J. Interictal high-frequency oscillations (80$500 \mathrm{~Hz})$ are an indicator of seizure onset areas independent of spikes in the human epileptic brain. Epilepsia. 2008; 49:1893-1907. [PubMed: 18479382]

Jaffe DB, Gutiérrez R. Mossy fiber synaptic transmission: communication from the dentate gyrus to area CA3. Prog Brain Res. 2007; 163:109-132. [PubMed: 17765714]

Jefferys JG, de la Prida LM, Wendling F, Bragin A, Avoli M, Timofeev I, Lopes da Silva FH. Mechanisms of physiological and epileptic HFO generation. Prog Neurobiol. Mar 7.2012 Epub ahead of print.

Kuriscak E, Marsalek P, Stroffek J, Wünsch Z. The effect of neural noise on spike time precision in a detailed CA3 neuron model. Comput Math Methods Med. 2012

Lalic T, Pettingill P, Vincent A, Capogna M. Human limbic encephalitis serum enhances hippocampal mossy fiber-CA3 pyramidal cell synaptic transmission. Epilepsia. 2011; 52:121-131. [PubMed: 21054347] 
Le Van Quyen M, Bragin A, Staba R, Crépon B, Wilson CL, Engel J Jr. Cell type-specific firing during ripple oscillations in the hippocampal formation of humans. J Neurosci. 2008; 28:61046110. [PubMed: 18550752]

Lopantsev V, Tempel BL, Schwartzkroin PA. Hyperexcitability of CA3 pyramidal cells in mice lacking the potassium channel subunit Kv1 1. Epilepsia. 2003; 44:1506-1512. [PubMed: 14636320]

Maier N, Güldenagel M, Söhl G, et al. Reduction of high-frequency network oscillations (ripples) and pathological network discharges in hippocampal slices from connexin 36-deficient mice. J Physiol. 2002; 541:521-528. [PubMed: 12042356]

Maier N, Nimmrich V, Draguhn A. Cellular and network mechanisms underlying spontaneous sharp wave-ripple complexes in mouse hippocampal slices. J Physiol. 2003; 550:873-887. [PubMed: 12807984]

Marcelin B, Chauvière L, Becker A, Migliore M, Esclapez M, Bernard C. h channel-dependent deficit of theta oscillation resonance and phase shift in temporal lobe epilepsy. Neurobiol Dis. 2009; 33:436-447. [PubMed: 19135151]

McKay BE, Molineux ML, Mehaffey WH, Turner RW. Kv1 K ${ }^{+}$channels control purkinjie cell output to facilitate postsynaptic rebound discharge in deep cerebellar neurons. J Neurosci. 2005; 25:1481-1492. [PubMed: 15703402]

Menegola M, Clark E, Trimmer JS. The importance of immunohistochemical analyses in evaluating the phenotype of Kv channel knockout mice. Epilepsia. 2012; 53(Suppl 1):142-149. [PubMed: 22612819]

Nakashiba T, Buhl DL, McHugh TJ, Tonegawa S. Hippocampal CA3 output is crucial for rippleassociated reactivation and consolidation of memory. Neuron. 2009; 25(62):781-787. [PubMed: 19555647]

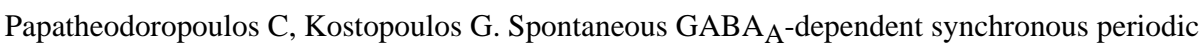
activity in adult rat ventral hippocampal slices. Neurosci Lett. 2002; 319:17-20. [PubMed: 11814643]

Prince DA, Connors BW. Mechanisms of interictal epileptogenesis. Adv Neurol. 1986; 44:275-299. [PubMed: 3518347]

Rex CS, Colgin LL, Jia Y, et al. Origins of an intrinsic hippocampal EEG pattern. PLoS One. 2009; 4:e7761. [PubMed: 19907647]

Rho JM, Szot P, Tempel BL, Schwartzkroin PA. Developmental seizure susceptibility of $\mathrm{K}_{\mathrm{V}} 1.1$ potassium channel knockout mice. Dev Neurosci. 1999; 21:320-327. [PubMed: 10575255]

Richichi C, Brewster AL, Bender RA, Simeone TA, Zha Q, Yin HZ, Weiss JH, Baram TZ. Mechanisms of seizure-induced 'transcriptional channelopathy' of hyperpolarization-activated cyclic nucleotide gated (HCN) channels. Neurobiol Dis. 2008; 29:297-305. [PubMed: 17964174]

Shu Y, Yu Y, Yang J, McCormick DA. Selective control of cortical axonal spikes by a slowly inactivating K+ current. Proc Natl Acad Sci U S A. 2007; 104:11453-11458. [PubMed: 17581873]

Simeone TA, Rho JM, Baram TZ. Single channel properties of hyperpolarization-activated cation currents in acutely dissociated rat hippocampal neurones. J Physiol. 2005; 568:371-380. [PubMed: 16123099]

Simeone TA, Wilcox KS, White HS. Subunit selectivity of topiramate modulation of heteromeric GABA(A) receptors. Neuropharmacology. 2006; 50:845-857. [PubMed: 16490221]

Simeone KA, Sabesan S, Kim do Y, Kerrigan JF, Rho JM, Simeone TA. L-Type calcium channel blockade reduces network activity in human epileptic hypothalamic hamartoma tissue. Epilepsia. 2011a; 52:531-540. [PubMed: 21269296]

Simeone TA, Wilcox KS, White HS. cAMP-dependent protein kinase A activity modulates topiramate potentiation of GABA(A) receptors. Epilepsy Res. 2011b; 96:176-179. [PubMed: 21665439]

Simeone TA, Wilcox KS, White HS. Topiramate modulation of $\beta(1)$ - and $\beta$ (3)-homomeric GABA(A) receptors. Pharmacol Res. 2011c; 64:44-52. [PubMed: 21421049]

Sloviter RS. Possible functional consequences of synaptic reorganization in the dentate gyrus of kainate-treated rats. Neurosci Lett. 1992; 137:91-96. [PubMed: 1625822] 
Smart SL, Lopantsev V, Zhang CL, et al. Deletion of the K(v)1.1 potassium channel causes epilepsy in mice. Neuron. 1998; 20:809-819. [PubMed: 9581771]

Spampanato J, Mody I. Spike timing of lacunosom-moleculare targeting interneurons and CA3 pyramidal cells during high-frequency network oscillations in vitro. J Neurophysiol. 2007; 98:96104. [PubMed: 17475718]

Staba RJ, Wilson CL, Bragin A, Jhung D, Fried I, Engel J Jr. High-frequency oscillations recorded in human medial temporal lobe during sleep. Ann Neurol. 2004; 56:108-115. [PubMed: 15236407]

Traub RD, Draguhn A, Whittington MA, Baldeweg T, Bibbig A, Buhl EH, Schmitz D. Axonal gap junctions between principal neurons: a novel source of network oscillations, and perhaps epileptogenesis. Rev Neurosci. 2002; 13:1-30. [PubMed: 12013024]

Treviño M, Vivar C, Gutiérrez R. Beta/gamma oscillatory activity in the CA3 hippocampal area is depressed by aberrant GABAergic transmission from the dentate gyrus after seizures. J Neurosci. 2007; 27:251-259. [PubMed: 17202493]

Urrestarazu E, Chander R, Dubeau F, Gotman J. Interictal high-frequency oscillations (100-500 Hz) in the intracerebral EEG of epileptic patients. Brain. 2007; 130:2354-2366. [PubMed: 17626037]

Wang H, Kunkel DD, Schwartzkroin PA, Tempel BL. Localization of $\mathrm{K}_{\mathrm{V}} 1.1$ and Kv1.2, two K channel proteins, to synaptic terminals, somata, and dendrites in the mouse brain. J Neurosci. 1994; 14:4588-4599. [PubMed: 8046438]

Wenzel HJ, Vacher H, Clark E, et al. Structural consequences of Kcna1 gene deletion and transfer in the mouse hippocampus. Epilepsia. 2007; 48:2023-2046. [PubMed: 17651419]

Wu C, Wah PL, Gillis J, et al. Size does matter: generation of intrinsic network rhythms in thick mouse hippocampal slices. J Neurophysiol. 2005; 93:2303-2317.

Wu K, Leung LS. Enhanced but fragile inhibition in the dentate gyrus in vivo in the kainic acid model of temporal lobe epilepsy: a study using current source density analysis. Neuroscience. 2001; 104:379-396. [PubMed: 11377842]

Zhou YD, Lee S, Jin Z, et al. Arrested maturation of excitatory synapses in autosomal dominant lateral temporal lobe epilepsy. Nat Med. 2009; 15:1208-1214. [PubMed: 19701204]

Zuberi SM, Eunson LH, Spauschus A, et al. A novel mutation in the human voltate-gated potassium channel gene $\left(\mathrm{K}_{\mathrm{V}} 1.1\right)$ associates with episodic ataxia type I and sometimes with partial epilepsy. Brain. 1999; 122:817-825. [PubMed: 10355668]

Zucker RS, Regehr WG. Short-term synaptic plasticity. Annu Rev Physiol. 2002; 64:355-405. [PubMed: 11826273] 


\section{Highlights}

1. In vitro hippocampal networks lacking $\mathrm{K}_{\mathrm{v}} 1.1$ generate sharp wave-high frequency oscillation complexes with pathologic characteristics.

2. Kcna1-null CA3 has enhanced coupling of excitatory inputs and population spike generation.

3. Kcna1-null CA3 principal cells have reduced spike timing precision.

4. Afferent inputs into Kcnal-null CA3 modulate pathologic sharp waves, high frequency oscillations and spike timing.

5. The Kcna1-null mossy fibers and medial perforant path axons are hyperexcitable and have reduced paired-pulse ratios.

6. In vitro pharmacological inhibition of $\mathrm{K}_{\mathrm{v}} 1.1$ recapitulates the pathologic oscillatory phenotype. 
A

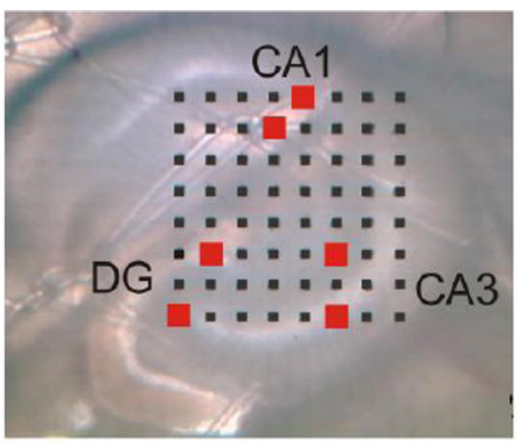

B

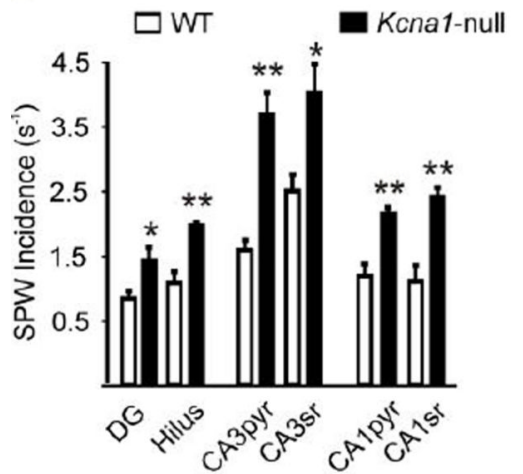

C

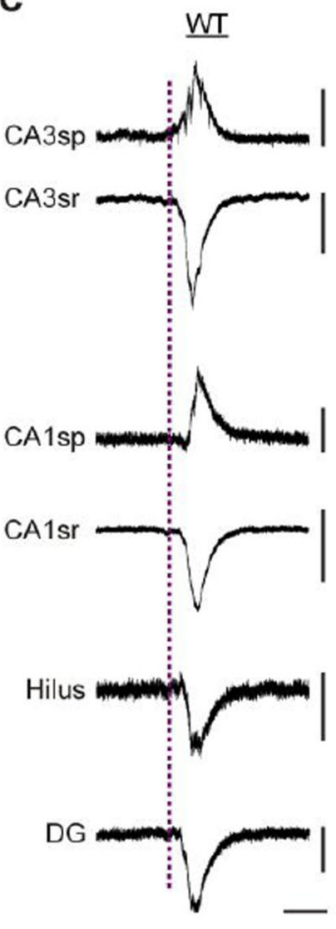

D

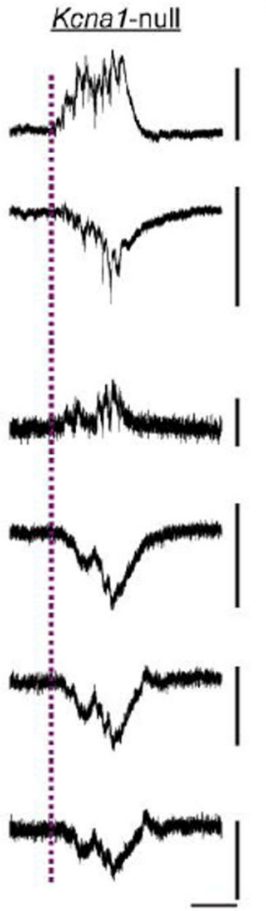

SPW Spatial-Temporal Propagation

WT

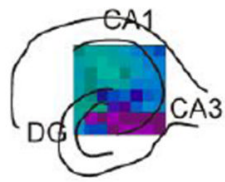

Kcna1null

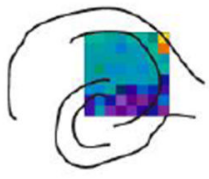

WT

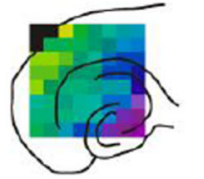

Kcna1-

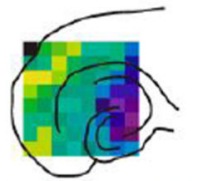

| IIIIIIIII||IIIIIIII||IIIIIIII|

$\begin{array}{llll}0 & 10 & 20 & 30\end{array}$

Figure 1. Kcnal-null SPWs have a higher incidence rate throughout the hippocampus and are longer in duration

(A) A digital image of a Kcna1-null (KO) hippocampal slice on an $8 \times 8$ multi-electrode array comprised of $50 \times 50 \mu \mathrm{m}$ electrodes and an inter-polar distance of $150 \mu \mathrm{m}$. (B) Left bar graph, Hippocampal slices from KO $(n=20)$ exhibit an increased SPW incidence rate in all regions compared to wild-type (WT) slices $(\mathrm{n}=15)$. Right bar graph, SPW duration measured in the CA3sr region is increased in $\mathrm{KO}(\mathrm{n}=9)$ compared to WT slices $(\mathrm{n}=11)$. (C) Representative SPWs from a WT and a KO slice from the corresponding hippocampal regions indicated by the red boxes in A. Calibration bars: x-ordinates are $50 \mathrm{~ms}$; all y-ordinates are $50 \mu \mathrm{V}$ except for WT and CA3sp $(100 \mu \mathrm{V})$, WT CA3sr $(200 \mu \mathrm{V})$, KO CA1sp $(20 \mu \mathrm{V})$, and WT CA1sr $(200 \mu \mathrm{V})$. (D) Representative examples of spatial-temporal propagation demonstrating SPWs are initiated in CA3 in WT and KO slices and spread with similar time-courses. The top two illustrations depict SPW propagation in hippocampi using a multielectrode array with inter-electrode spacing of $150 \mu \mathrm{m}$. The bottom two illustrations demonstrate propagation into the entorhinal cortex recorded with inter-electrode spacing of $300 \mu \mathrm{m}$. $* \mathrm{p}<0.05 ; * * \mathrm{p}<0.001$. CA, cornus ammonis; DG, dentate gyrus; sg, sp, sr, strata granulosum, pyramidale and radiatum, respectively. 
A

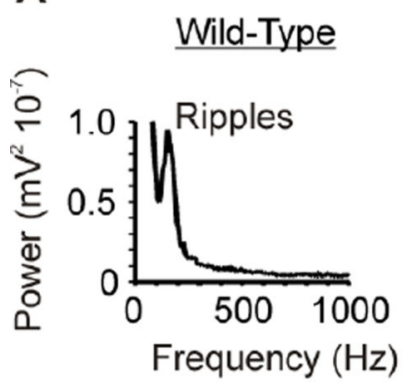

B

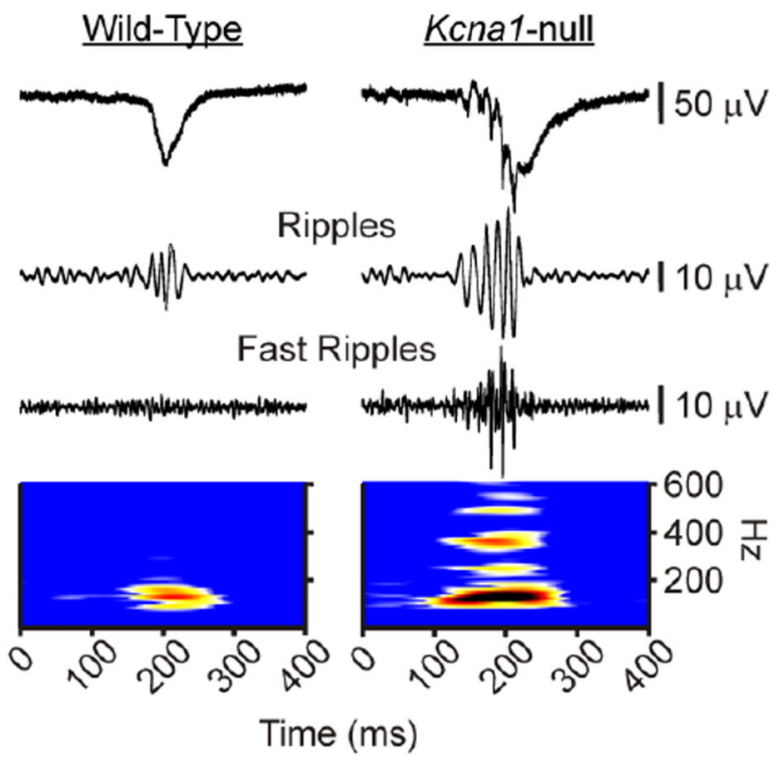

C
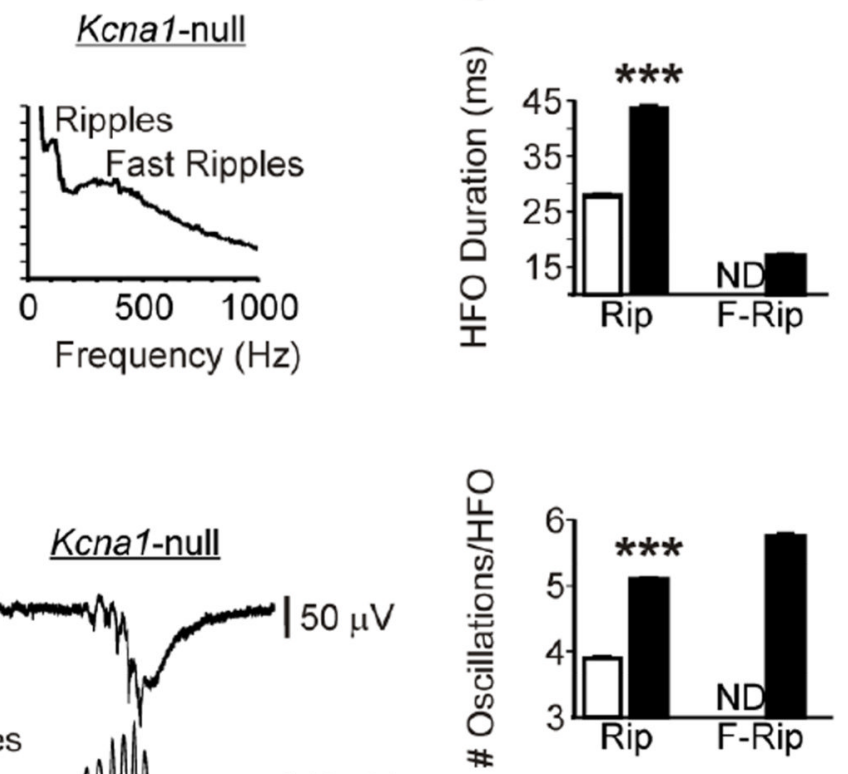

D HFO Spatial Presence
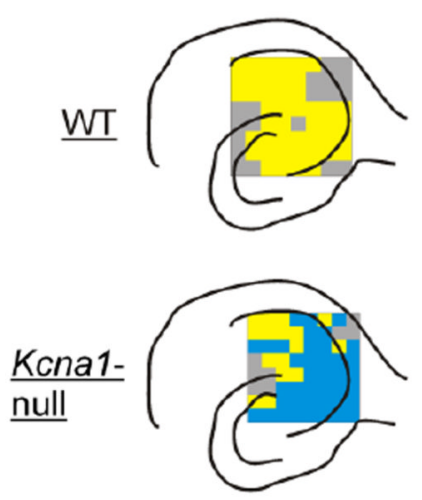

WI
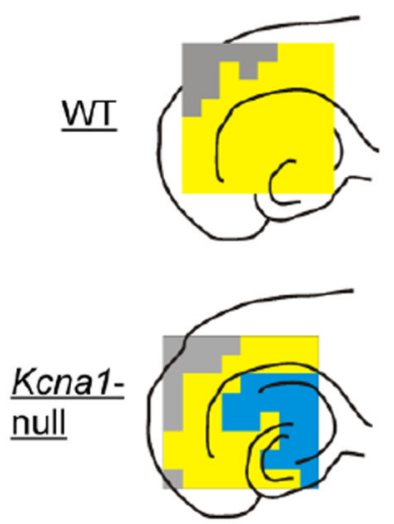

Rip

Rip and F-Rip

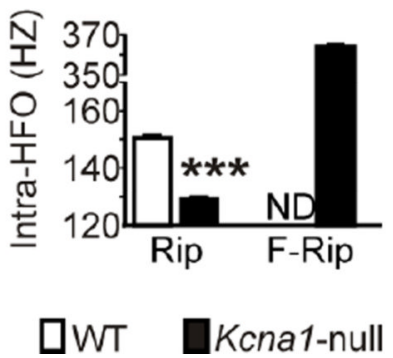

ND

Figure 2. Pathologic HFOs are apparent throughout the Kcna1-null hippocampus

(A) Power spectra of SPWs in the CA3sr illustrating the sharp ripple peak present in both

WT and KO hippocampal slices, whereas the broad fast ripple peak appears only in the KO spectrum. (B) Representative SPWs from the CA3sr region of a WT and a KO slice filtered to demonstrate the presence/absence of high frequency oscillations (HFOs), specifically ripples at 100-175 Hz and fast ripples at 200-600 Hz. Lower panel, Time-frequency representation of the component frequencies of the SPWs band-pass filtered between 100$600 \mathrm{~Hz}$. (C) Ripple duration and cycles per ripple are increased, whereas intra-ripple frequency is decreased in KO slices $(n=11)$ compared to WT slices $(n=11)$. Fast ripple durations, cycles and intra-fast ripple frequency are also presented for KO slices, but are not detectable (ND) in WT slices. ***p<0.001. (D) Representative illustrations of spatial maps of the presence of ripples and fast ripples for individual slices. The presence of HFOs was determined by generating power spectra and time-frequency representations of five-ten minute recordings from each electrode. Ripples are widespread in hippocampi of both genotypes. Fast ripples are prevalent only in KO hippocampi and do not spread into the entorhinal cortex. We performed similar spatial maps of HFOs for 3-5 slices per microelectrode array size for each genotype and always found similar patterns as those presented. 
A

A
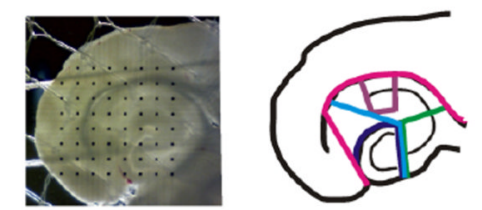

Prep\#1 - separates EC and hippo Prep\#2 - isolates dentate gyrus Prep\#3 - isolates CA3 and CA1 Prep\#4 - isolates CA3 Prep\#5 - isolates CA1
B

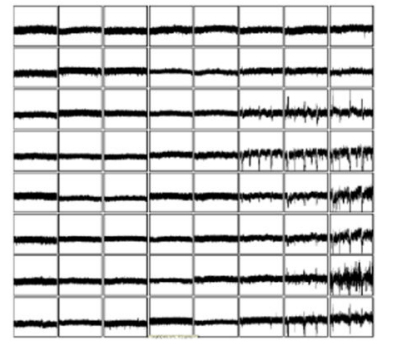

Separated HEC

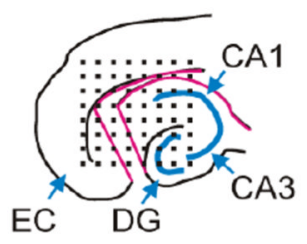

C

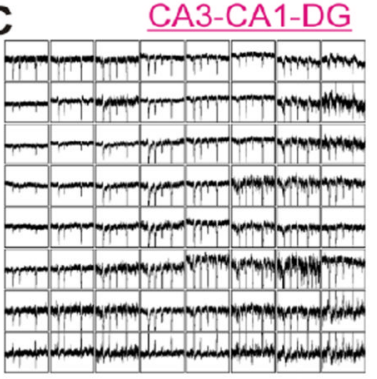

E

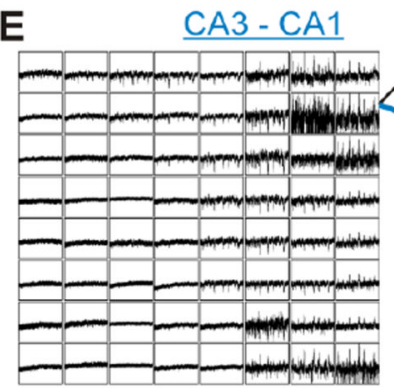

G

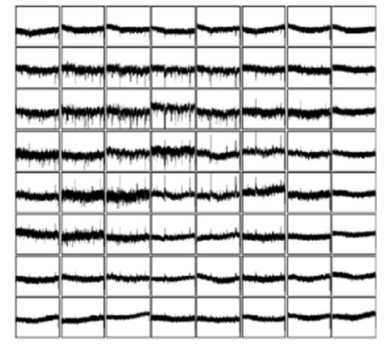

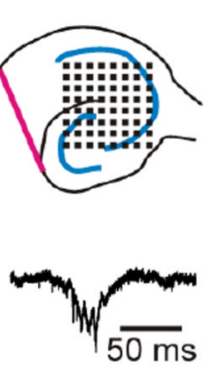

D

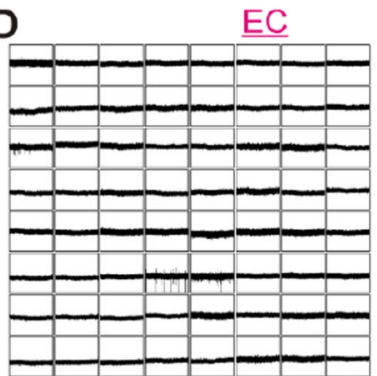

$\underline{D G}$

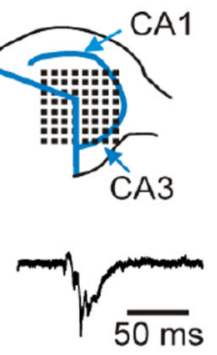

F
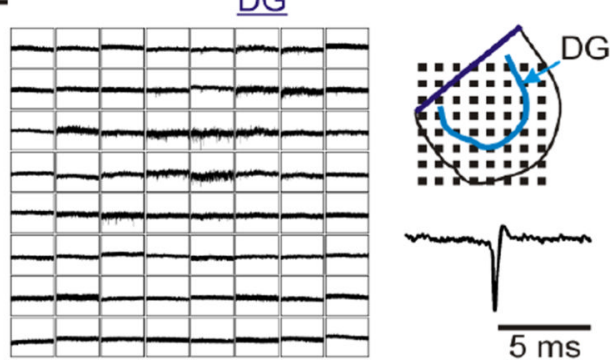

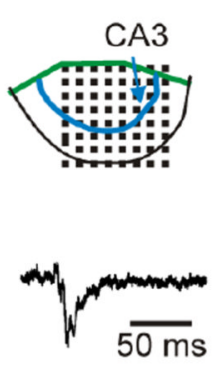

H

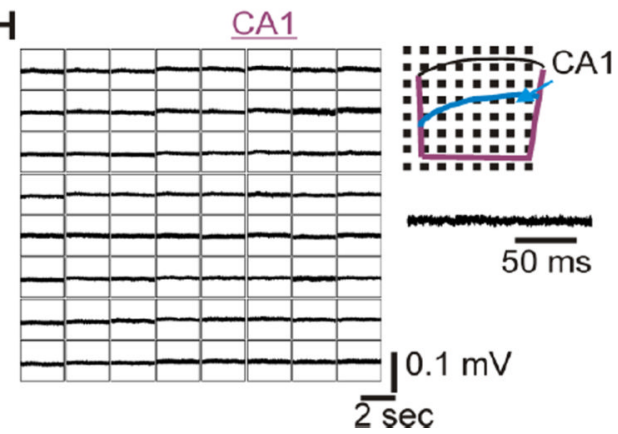

Figure 3. Kcna1-null SPW-HFO activity persists only in CA3-containing mini-slices (A) Digital image of a hippocampal-entorhinal cortex (HEC) slice on a microelectrode array with $300 \mu \mathrm{m}$ inter-electrode spacing and an illustration of the knife cuts used to isolate subregions from an HEC slice. (B) A separated hippocampus and entorhinal cortex (EC) on the same $300 \mu \mathrm{m}$ array. SPW-HFO activity is only present in the hippocampus, whereas occasional single units are observed in the EC and presubiculum. (C) An isolated hippocampus (CA3-CA1-DG) on a $150 \mu \mathrm{m}$ array with robust SPW activity. (D) When EC is isolated on a $300 \mu \mathrm{m}$ array, spontaneous single units are present in the presubiculum and EC. (E) SPWs are present in mini-slices of isolated CA3-CA1 (150 $\mu \mathrm{m}$ array). (F) SPWs are absent in isolated dentate gyrus (DG; $150 \mu \mathrm{m}$ array), but spontaneous single units often 
occur in the hilar region. (G) The isolated CA3 mini-slice is able to support the generation of SPW activity (150 $\mu \mathrm{m}$ array). (H) Isolated CA1 lacks spontaneous activity (150 $\mu \mathrm{m}$ array). $\mathrm{N}=3-4$ slices per micro-dissection. 
A

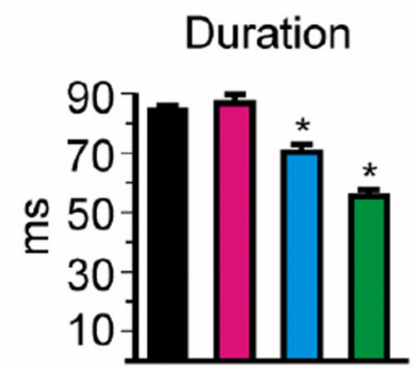

B

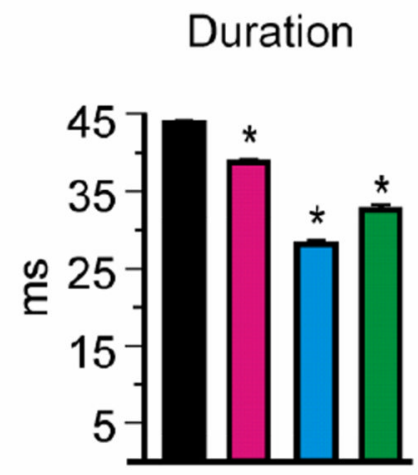

$\underline{\text { SPW }}$

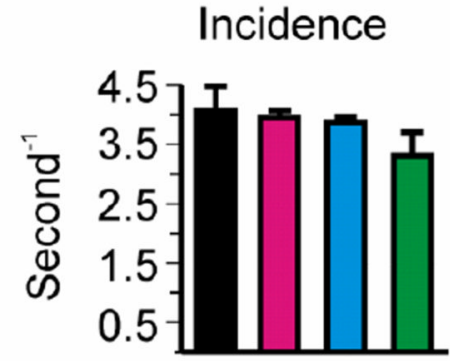

Ripple

Intra-HFO Frequency

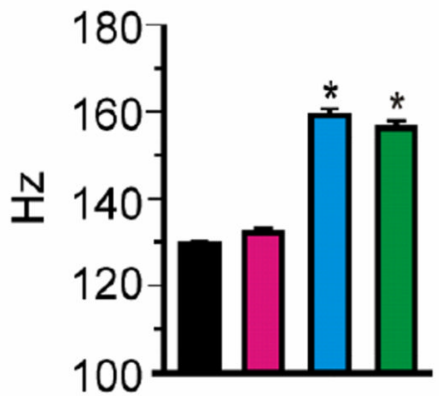

\section{Fast Ripple}
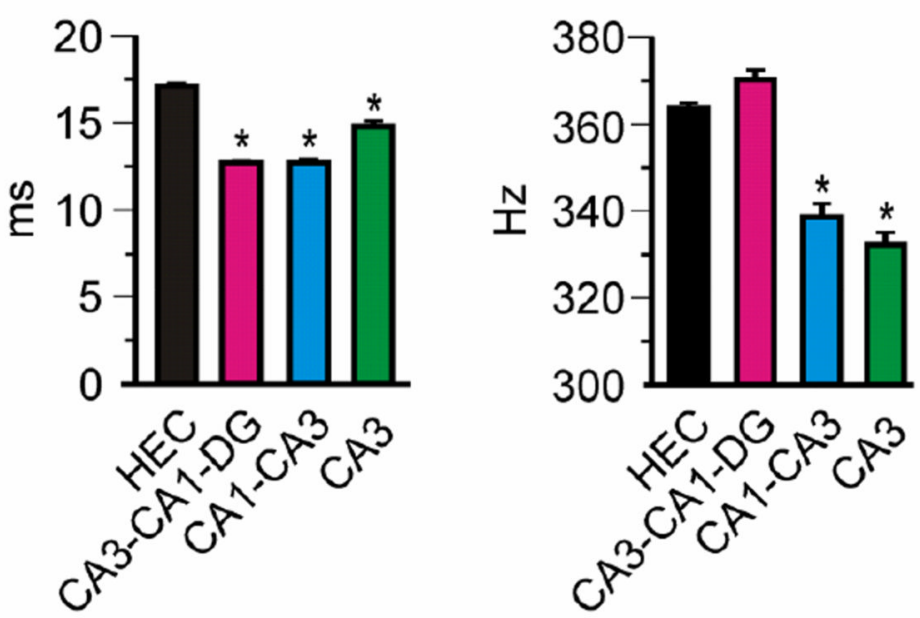

Figure 4. Afferent inputs determine SPW and HFO durations, and HFO frequencies Quantification of KO CA3sr SPW-HFO characteristics from intact HEC slices, isolated hippocampus (CA3-CA1-DG), isolated CA1-CA3 region, and isolated CA3. (A) Removal of the EC, DG and CA1 reduces the duration of SPWs, however, the incidence rates do not differ among the dissected slices. (B) Ripple and fast ripple durations decrease with removal of the EC, DG and CA1. In mini-slices of CA1-CA3 and CA3, the intra-HFO frequencies increase for ripples and decrease for fast ripples. Statistical significance was calculated with a one-way ANOVA and Tukey's multiple comparisons post-test : ${ }^{*} \mathrm{p}<0.001 ; \mathrm{n}=3-4$ slices per micro-dissection. 


\section{A Single Unit Classification}
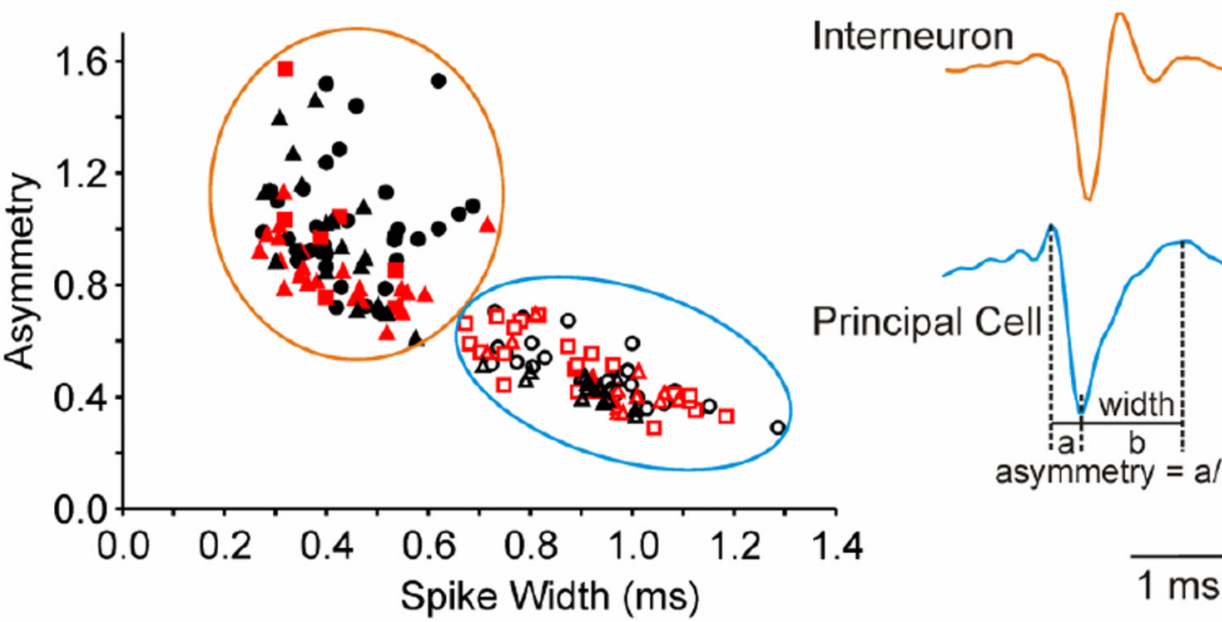

Autocorrelation

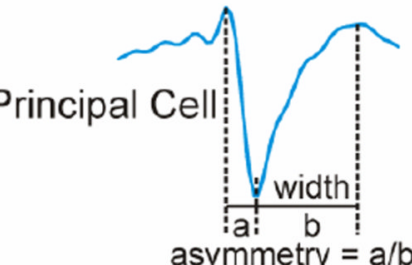

asymmetry $=a / b$

$1 \mathrm{~ms}$

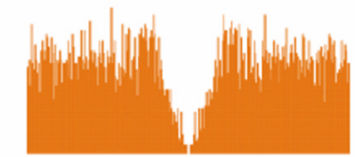

B $\underline{\text { WT }}$

$\underline{\mathrm{KO}}$

KO-CA3

C

Doublet Spikes

D

minislice
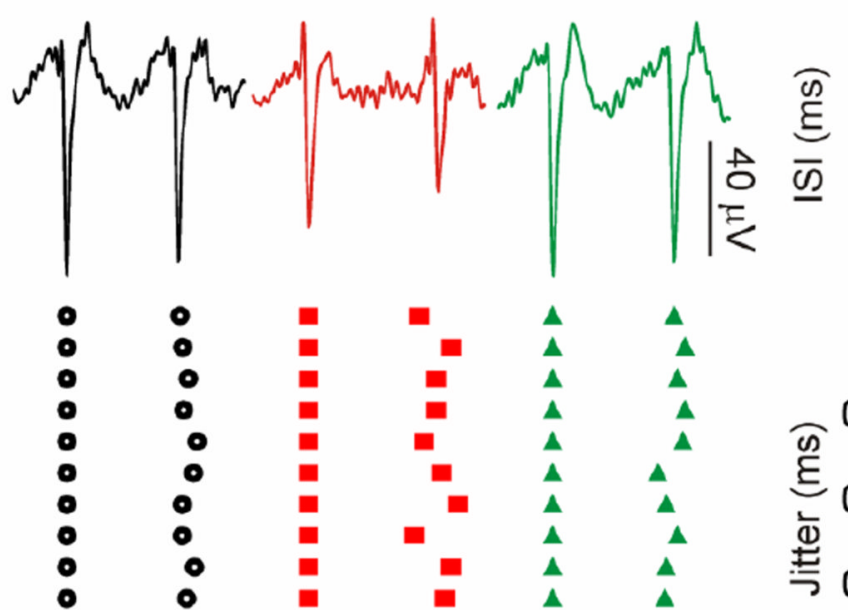
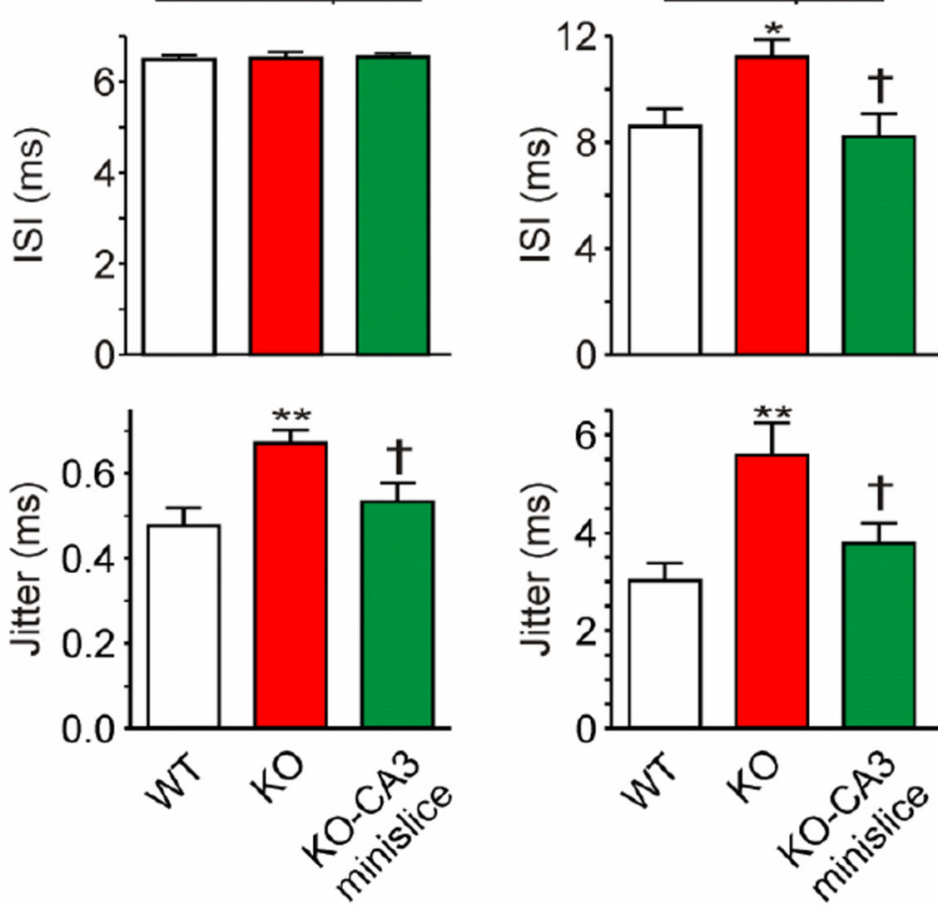

Figure 5. Kcna1 null CA3 principal cells have increased spike timing jitter

Spontaneous unit firing was identified in recordings from electrodes underlying the CA3 principal cell layer. Units were grouped using principal component analysis. (A) Single units were classified as interneurons or principal cells according to spike-width, asymmetry and the autocorrelation (see Table 1 for quantification). Interneurons, open symbols. Principal cells, closed symbols. WT, black circles. KO, red squares. KO-CA3 mini-slice, red triangles. WT + dendrotoxin-k, black triangles. (B) Representative traces and timing of successive doublet spikes illustrate the increase in variability of individual KO principal cell ISIs, which is reduced upon isolation of the CA3 region. (C) Quantification of the inter-spike interval (ISI) and jitter for doublets between SPWs ( $\mathrm{n}=8 \mathrm{WT}, 9 \mathrm{KO}, 10$ KO-CA3 neurons). 
(D) Quantification of the inter-spike interval (ISI) and jitter of single units during SPWs ( $\mathrm{n}=$ 19 WT, 17 KO, 13 KO-CA3 neurons). Statistical significance determined with a one-way ANOVA and Tukey's multiple comparisons post-test (compared to WT, ${ }^{*} \mathrm{p}<0.05$, ${ }^{*} \mathrm{p}<0.01$; compared to $\mathrm{KO}, \dagger \mathrm{p}<0.05)$. 
A
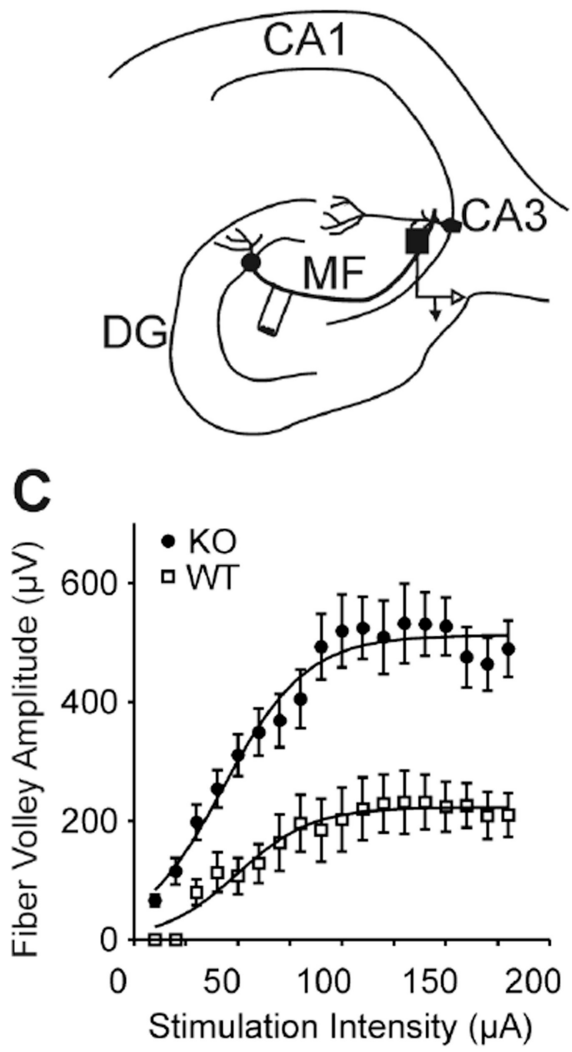

E

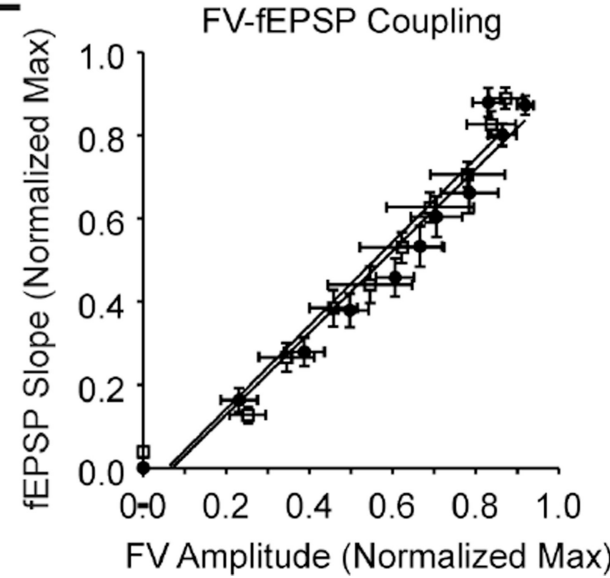

B
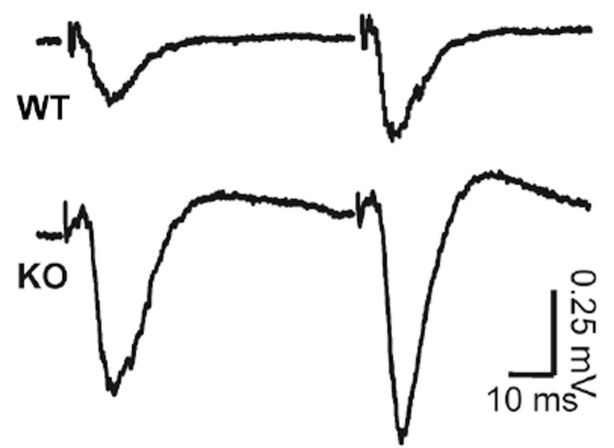

D

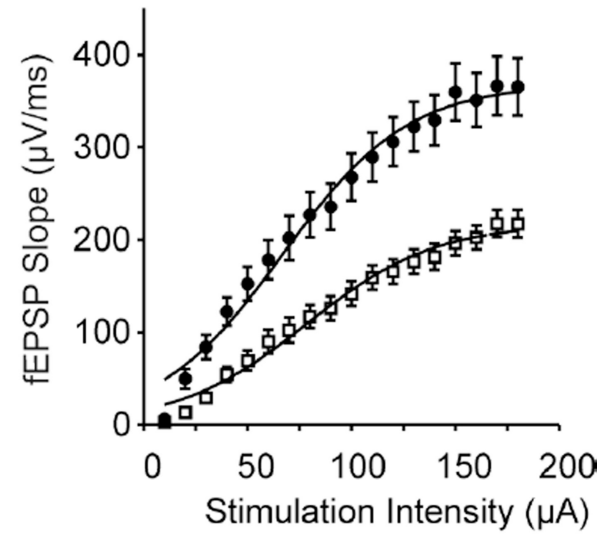

$\mathbf{F}$

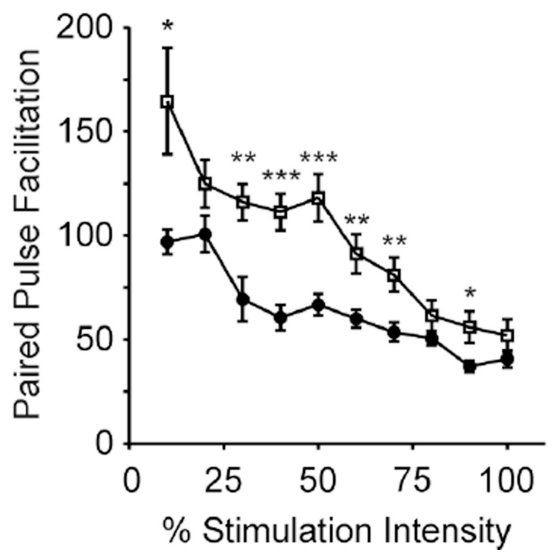

Figure 6. Kcna1-null mossy fiber-CA3 synapses are hyperexcitable and have reduced pairedpulse ratios

(A) A simplified illustration of mossy fiber innervation of CA3 with the stimulation site in the hilar region of the dentate gyrus and recording electrode in the CA3 stratum lucidum indicated. (B) Representative traces of WT and KO field responses to mossy fiber paired pulse stimulations [50 ms ISI; half maximal stimulation intensity $\left(\mathrm{V}_{50}\right)$ ]. (C) KO fiber volley amplitudes were increased and $\mathrm{V}_{50}$ 's decreased (see Table 2) indicating greater recruitment per stimulation intensity of hyperexcitable axons ( $\mathrm{n}=3 \mathrm{WT}, 5 \mathrm{KO}$ slices). (D) Sequential increases in the stimulation intensity revealed increased slopes and lowered $\mathrm{V}_{50}$ 's for $\mathrm{KO}$ mossy fiber synapses (see Table 2) ( $\mathrm{n}=5 \mathrm{WT}, 7 \mathrm{KO}$ slices). (E) Pre-synaptic fiber volley 
(FV) coupling to the post-synaptic fEPSP does not differ between genotypes. (F) Paired stimulations (50 ms ISI) indicate a sustained decrease in KO paired pulse facilitation across intensities ( $\mathrm{n}=5 \mathrm{WT}, 7 \mathrm{KO})$. Statistical significance was calculated with a oneway ANOVA with repeated measures, ${ }^{*} \mathrm{p}<0.05 ; * * \mathrm{p}<0.01 ; * * * \mathrm{p}<0.001$. 
A
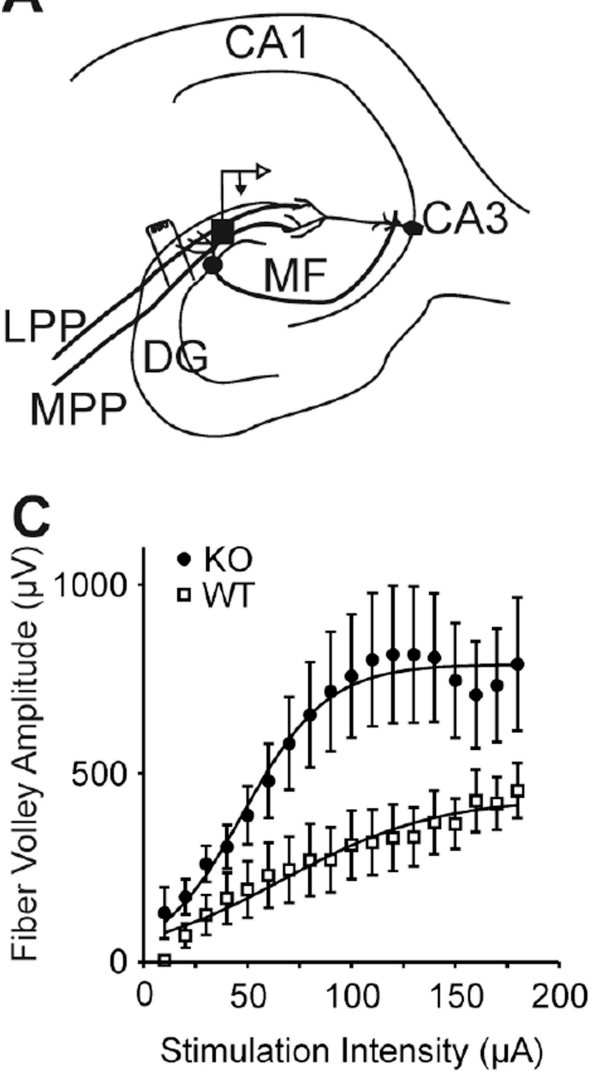

E

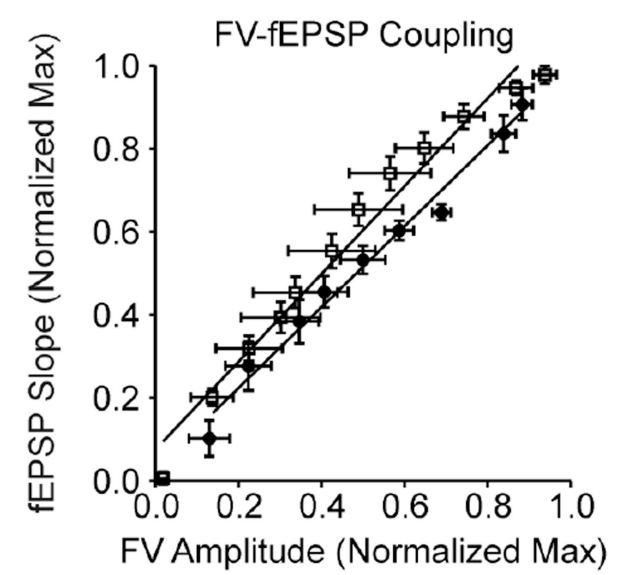

B
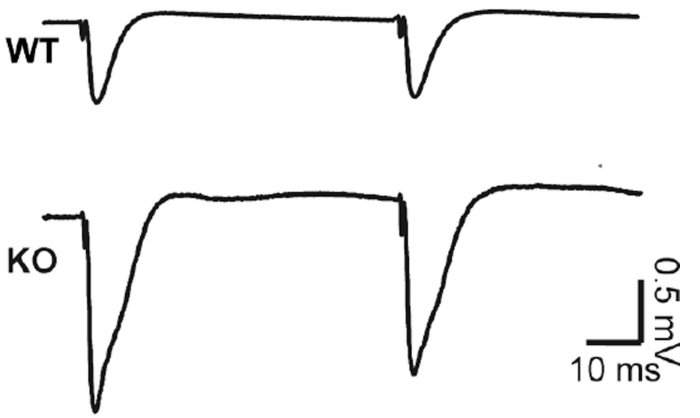

D

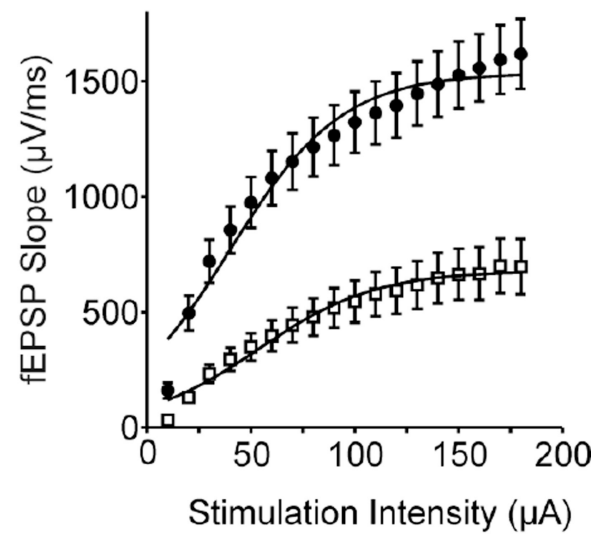

$\mathbf{F}$

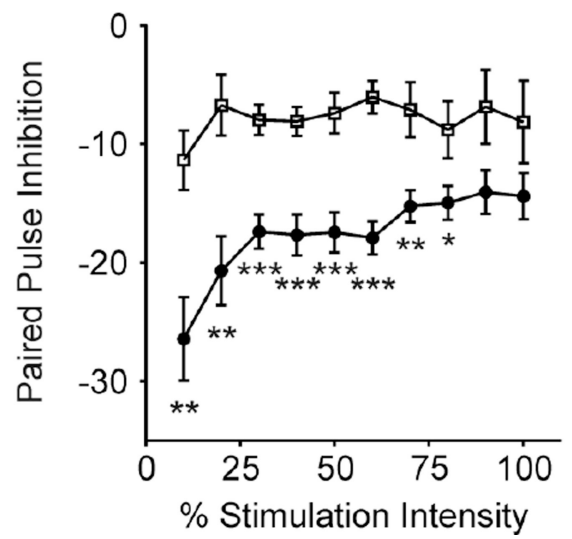

Figure 7. Kcnal-null MPP-DG synapses are hyperexcitable and have reduced paired pulse ratios (A) A simplified illustration of perforant path stimulation site and the nearby recording electrode in the dentate gyrus. (B) Representative traces of WT and KO field responses to MPP paired pulse stimulations [50 ms ISI; half maximal stimulation intensity $\left.\left(\mathrm{V}_{50}\right)\right]$. (C) $\mathrm{KO}$ fiber volley amplitudes were increased and $\mathrm{V}_{50}$ 's decreased (see Table 2 ) indicating hyperexcitable axons with greater recruitment per stimulation intensity ( $=6 \mathrm{WT}, 3 \mathrm{KO}$ slices). (D) Sequential increases in the stimulation intensity revealed increased slopes and lowered $\mathrm{V}_{50}$ 's for KO MPP-DG synapses (see Table 2) ( $\mathrm{n}=9 \mathrm{WT}, 12 \mathrm{KO}$ slices). (E) Presynaptic fiber volley (FV) coupling to the post-synaptic fEPSP is not different between genotypes. (F) Paired stimulations (50 ms ISI) indicated a sustained increase in KO paired 
pulse inhibition across stimulation intensities ( $\mathrm{n}=9 \mathrm{WT}, 12 \mathrm{KO}$ slices). Statistical significance was calculated with a oneway ANOVA with repeated measures, ${ }^{*} \mathrm{p}<0.05$; $* * \mathrm{p}<0.01 ; * * * \mathrm{p}<0.001$. 
A

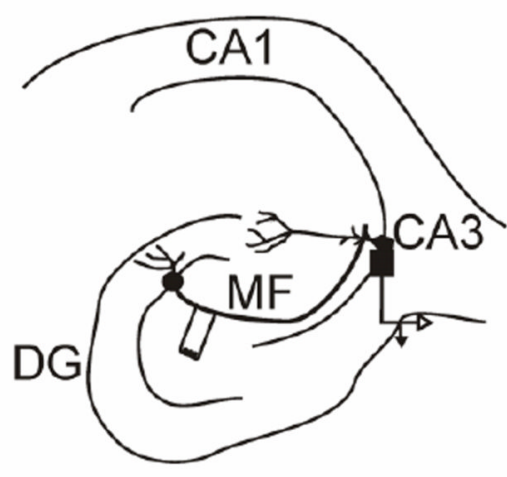

B

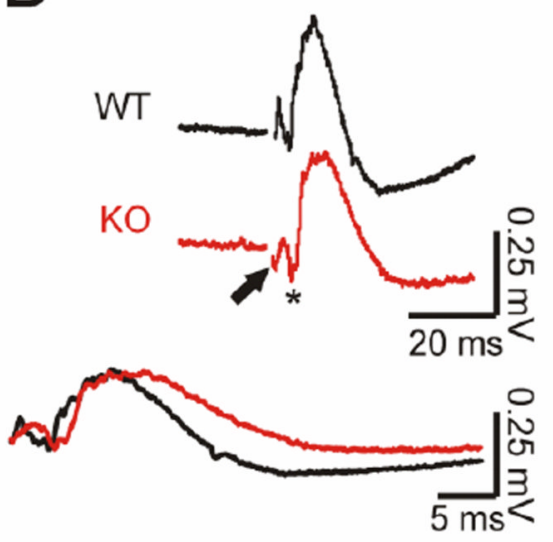

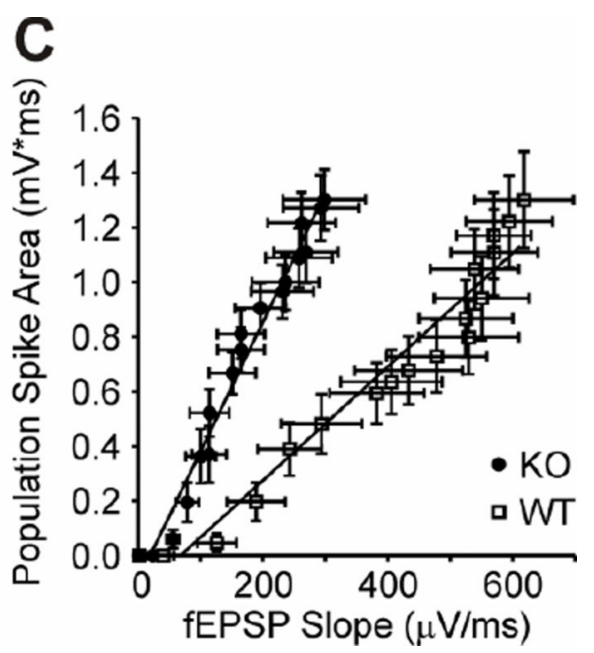

Figure 8. Kcna1 null CA3 has enhanced extracellular field EPSP-to-spike (E-S_coupling (A) A simplified illustration of mossy fiber innervation of CA3 indicating the stimulation site in the hilar region of the dentate gyrus and the recording electrode in the CA3 stratum pyramidale. (B) Representative traces of WT and KO population spikes in response to mossy fiber stimulation. Traces with similar population spikes were chosen to illustrate the differences in fEPSP slope. The fEPSP and population spike are indicated on the KO trace by an arrow and asterisks, respectively. Bottom, traces are overlaid to illustrate the difference of fEPSP slopes, but similarity of population spikes. (C) $K O \mathrm{E}-\mathrm{S}$ coupling is shifted to the left due to a decrease in the fEPSP slope indicating that a given fEPSP slope elicits a larger population spike in the $\mathrm{KO}(\mathrm{n}=7 \mathrm{WT}, 6 \mathrm{KO}$ slices). 
A

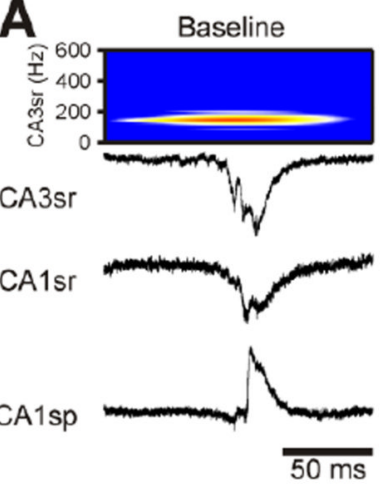

B
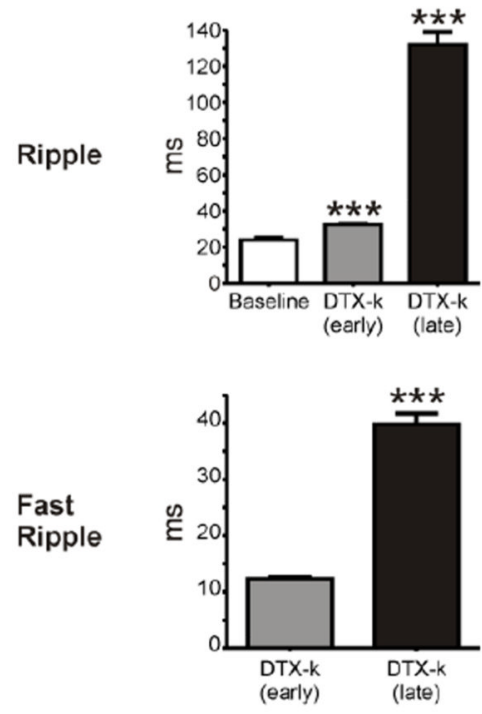

C

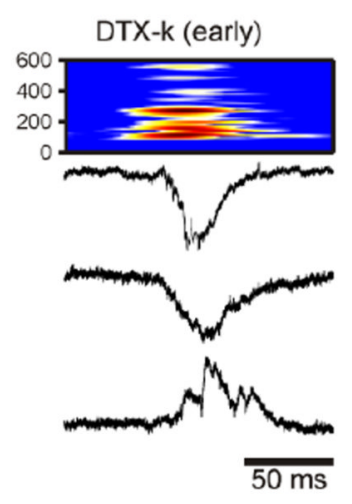

Intraburst Frequency
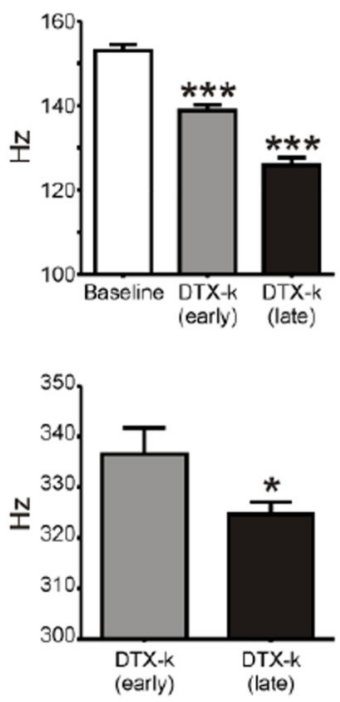

Incidence
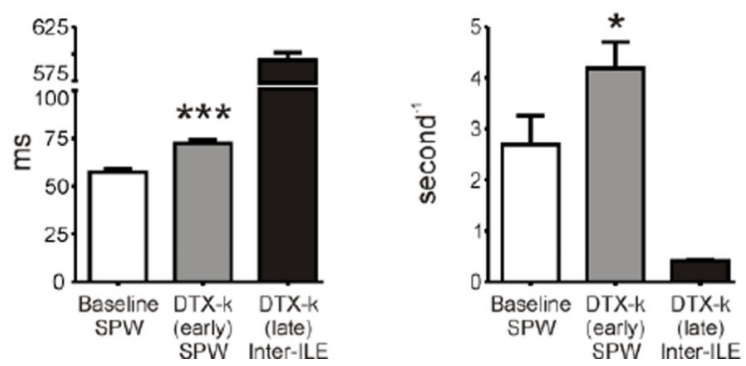

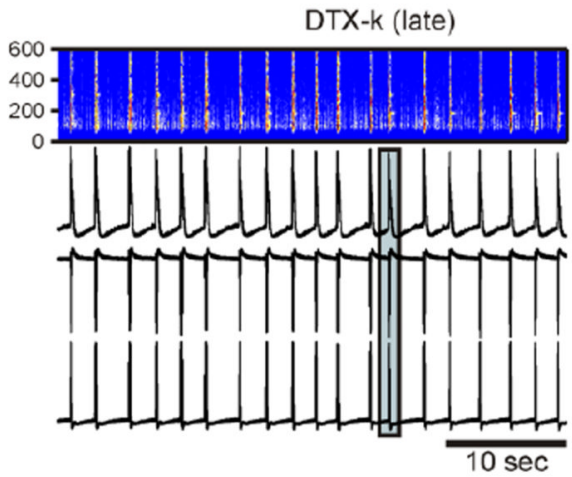

Doublet Spikes
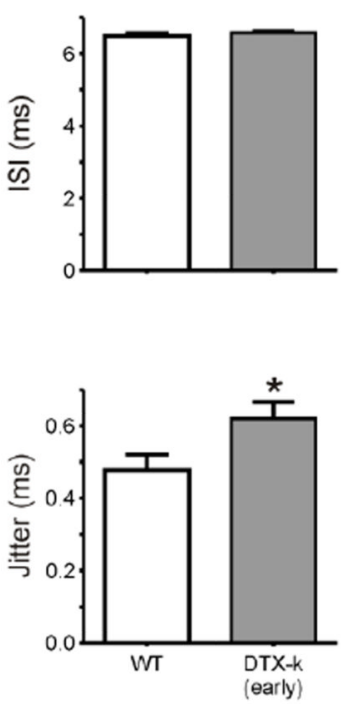

$\mathbf{E}$

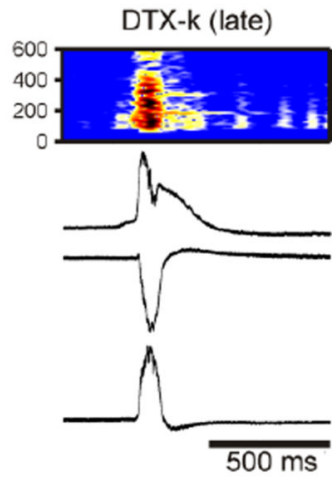

SPW Spikes
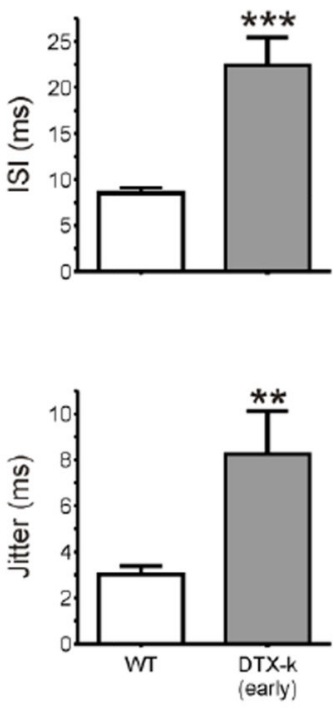

MPP-DG

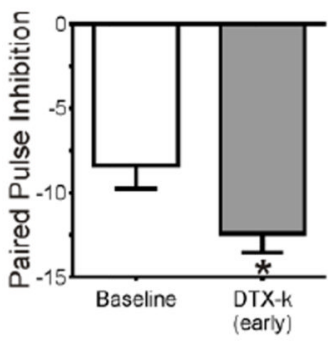

Figure 9. Pharmacological blockade of $K_{v} 1.1$ in wild-type hippocampal slices promotes pathologic oscillations

Perfusion of dendrotoxin- $\mathrm{\kappa}$ (DTX-k, $100 \mathrm{nM}$ ) onto wild-type slices induced two distinct phases of activity [increased SPW-HFOs and development of inter-ictal like events (interILE)]; therefore, duration and frequencies of SPW-HFOs or inter-ILE-HFOs were analyzed during an early (10-45 $\mathrm{min}$ ) and a late (once inter-ILEs were present $>40 \mathrm{~min}$ ) phase. (A) Representative SPWs from the CA3sr, CA1sr and CA1sp regions of a WT slice at baseline and during the early and late phases of DTX-k treatment. Time-frequency representations of the component frequencies of the CA3sr SPWs (band-pass filtered between 100-600 Hz) depict DTX-k treatment induced spectral disorganization. Middle panel: Note the significant 
increase in synchronicity and excitability during the late phase. Right panel: Magnification of the boxed, late phase inter-ILE from CA3sr, CA1sr and CA1sp. (B) Bar graphs depicting quantification of ripple and fast ripple durations, and intraburst frequencies ( $\mathrm{n}=5$ slices). (C) Quantification of SPW and inter-ILE duration and incidence during early and late phases of DTX-k treatment ( $n=5$ slices). (D) DTX-k increased the jitter of CA3 principal cell doublets between SPWs ( $\mathrm{n}=8 \mathrm{WT}, 11$ DTX-k neurons). The inter-spike interval (ISI) and jitter of spikes occurring during SPWs were increased by DTX-k $(n=19$ WT, 11 DTX-k neurons). (E) DTX-k reduced paired pulse facilitation (50 $\mathrm{ms}$ at the $50 \%$ stimulation intensity) of mossy fiber (MF)-CA3 synapses and increased paired pulse inhibition of medial perforant path (MPP)-dentate gyrus (DG) synapses. Statistical significance (compared to baseline, ${ }^{*} \mathrm{p}<0.05,{ }^{* * *} \mathrm{p}<0.001$ ) was determined with either an unpaired t-test or a one-way ANOVA with a Dunnett's post-test where appropriate. 


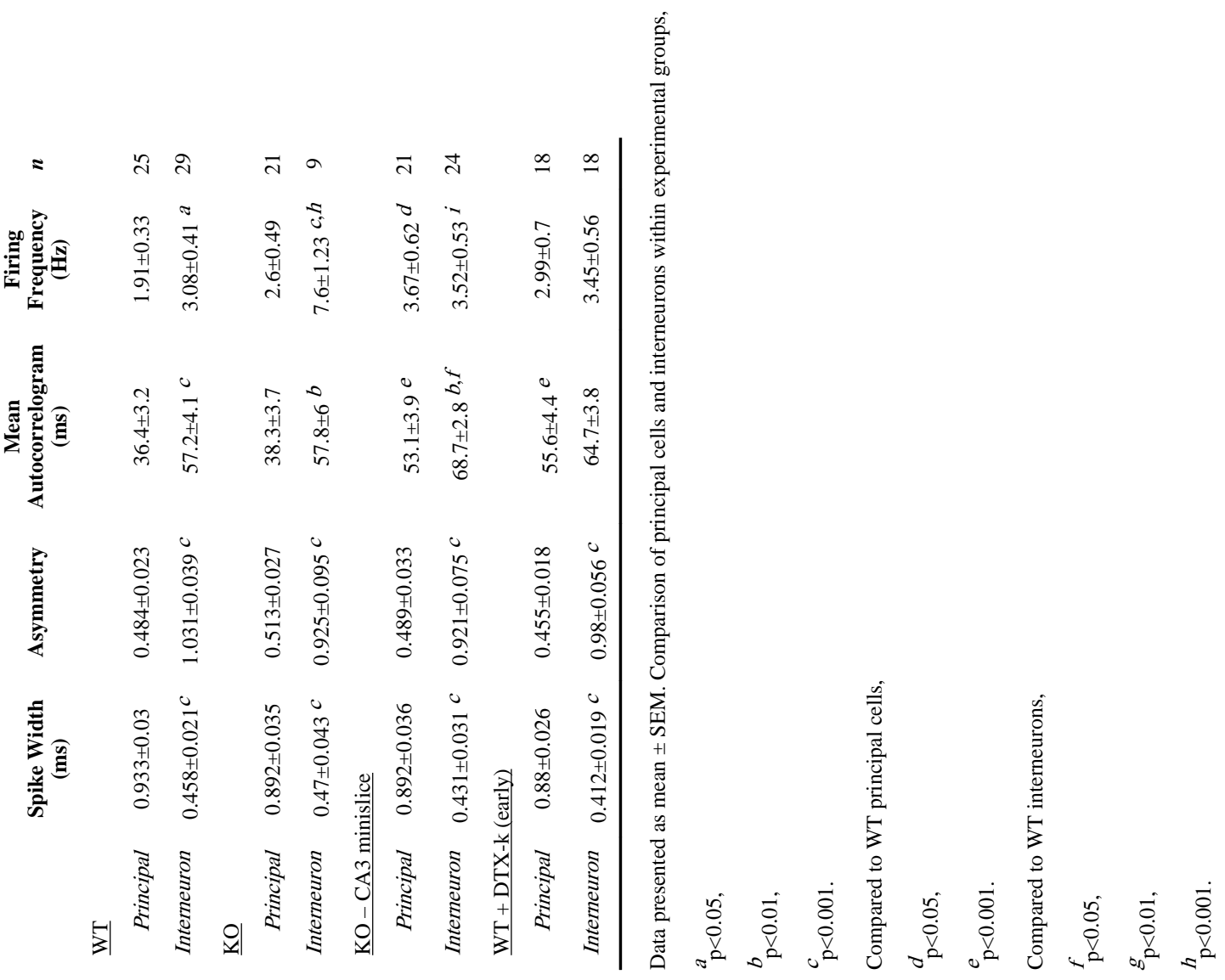

Neurobiol Dis. Author manuscript; available in PMC 2014 June 01. 


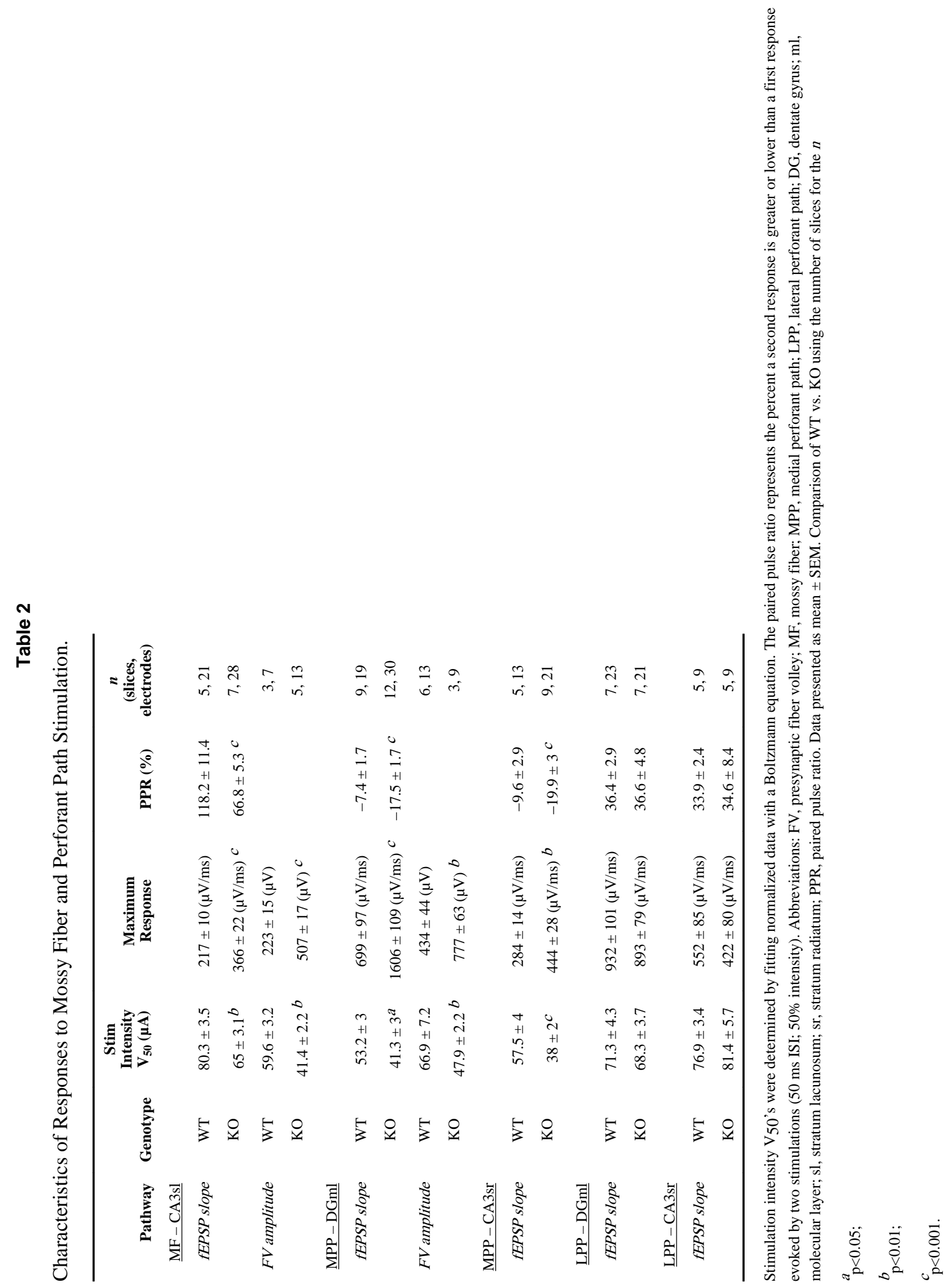

